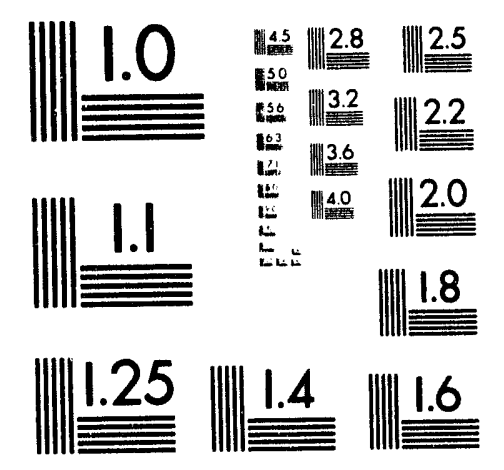



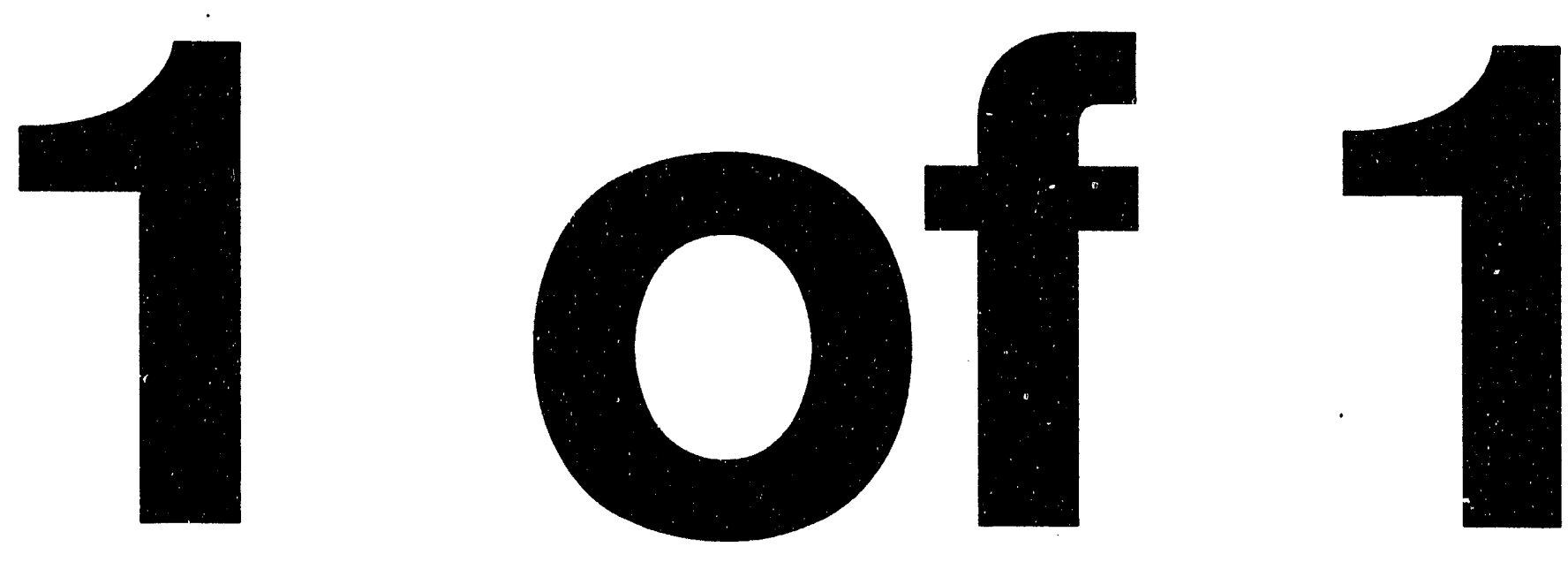


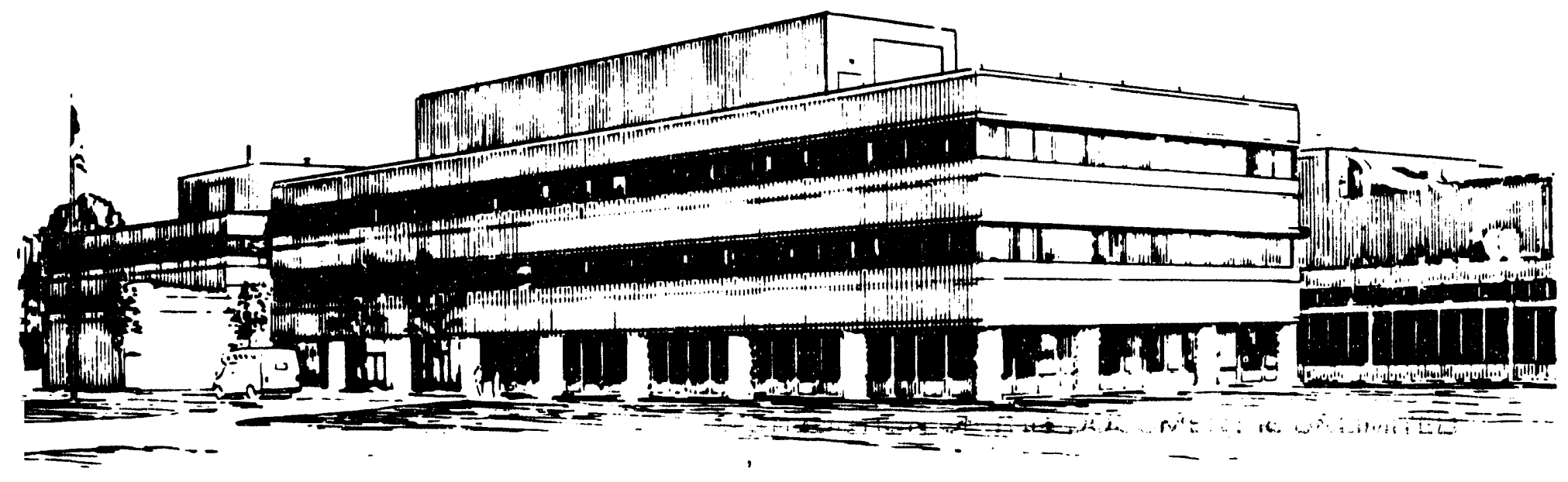




\section{NOTICE}

This report was prepared as an account of work sponsored by an agency of the United States Government. Neither the United States Government nor any agency thereof, nor any of their employees, makes any warranty, express or implied, or assumes any legal liability or responsibility for the accuracy, completeness, or usefulness of any information, apparatus, product, or process disclosed, or represents that its use would not infringe privately owned rights. Reference herein to any specific commercial produce, process, or service by trade name, trademark, manufacturer, or otherwise, does not necessarily censtitute or imply its endorsement, recommendation, or favoring by the United States Government or any agency thereof. The views and opinions of authors expressed herein do not necessarily state or reflect those of the United States Government or any agency thereof.

\section{NOTICE}

This report has been reproduced from the best available copy. Avallable in paper copy and microfiche.

Number of pages in this report: 53

DOE and DOE contractors can obtain copies of this report from:

Office of Scientific and Technical Information P.O. Box 62

Oak Ridge, TN 37831; (615) 576-8401.

This report is publicly available from the:

National Technical Information Service

Department of Commerce

5285 Port Royal Road

Springfield, Virginia 22161

(703) $487-4650$ 
August 16.1993

\title{
Studies of Local Electron Heat Transport on TFTR
}

\author{
E. D. Fredrickson, Z. Y. Chang: A. Janos, \\ K. M. McGuire, S. Scott, and G. Taylor \\ Princeton Plasma Physics Laboratory \\ Princeton, NJ 08543
}

\begin{abstract}
The anomalously fast relaxation of the perturbations to the electron temperature profile caused by a sawtooth crash has been studied extensively on TFTR. We will show that on a short timescale the heat pulse is not simply diffusive as has been generally assumed, but that modeling of the heat pulse requires a transient enhancement in $\chi_{e}$ following the sawtooth crash. It will be shown that the time-dependent enhancement in $\chi_{e}$ predicted by non-linear thermal transport models, i.e., incremental $\chi$ models or the Rebut-LalliaWatkins transport model, is much smaller than that required to explain the anomalies in the heat pulse propagation.
\end{abstract}




\section{Introduction}

The transport of thermal energy in tokamak plasmas has long been known to be larger than neoclassical calculations predict. It is commonly assumed that 'small' scale electrostatic turbulence, magnetic stochasticity or some similar mechanism is responsible for the enhanced transport. ${ }^{1}$ Studies of the propagation of heat pulses following sawtooth crashes started with the hope that this approach would vield a local measurement of the electron thermal diffusivity, independent of the uncertainties of power balance analysis, and which then might improve understanding of the nature of anomalous transport. ${ }^{2,3}$

When studies of sawtooth induced heat pulse propagation were undertaken on TFTR, ${ }^{4}$ it was found that the heat pulse propagation was not consistent with the estimates of thermal diffusivity, $\chi_{e}$ from power balance analysis. These results suggested several interesting possible explanations, including that the model of the sawtooth crash was incomplete or that the heat flux had a non-linear dependence on the temperature gradient. ${ }^{4.5}$ Recently there has been renewed interest in both of these possibilities. New experimental and theoretical evidence suggests that the sawtooth crash mechanism may transiently enhance the electron thermal conductivity in the region outside the mixing radius. ${ }^{6}$ The interest in 'incremental' or nonlinear models for $\chi_{e}$ is motivated in part by the long standing observation of the 'resilience' of the electron temperature profile to changes in the heating profile ${ }^{i-10}$ and more recently by JET results which have purported to find agreement between the electron thermal diffusivity determined from heat pulse analysis, $\chi^{H P}$, and the $\chi^{\text {inc }}$ determined from power balance analysis of parameter scans. ${ }^{11}$ However. in this paper it will be shown that $\chi^{H P}$ is several times larger than the $\chi^{\text {inc }}$ for TFTR data.

In this paper we will demonstrate that it is not possible, at least for TFTR data, to simulate the sawtooth induced heat pulses with a simple model for heat diffusion, thus $\chi^{H P}$ derived from sawtooth induced heat pulses is not a meaningful quantity. At small minor radii, in the radial range $r_{\text {maxi }}<$ $r<1.4 r_{m i x}$, the heat pulse propagation can be dominated by the ballistic effect. ${ }^{6}$ (Throughout this paper, $r_{m i x}$ will have the standard definition, i.e., the radius to which the sawtooth crash flatters the temperature profile.) At larger minor radius (later times) the ratio of signal-to-noise is lower and 
the sharp features present at smaller radii (shorter timescales) have largely relaxed with the result that the heat pulse shapes may be approximately simulated with many models, e.g., the options of varying the radial profile of $\chi^{\text {inc }}$ or $\chi$, introducing coupling to the density pulse ${ }^{12}$ or including an anomalous damping term ${ }^{13}$ allow considerable leewav for approximate fits to the experimental data. Thus, at neither small nor large minor radius is it possible to draw unambiguous conclusions about the nature of anomalous transport for sawtooth induced heat pulse studies.

In the first part of this paper we will discuss the limitations inherent in comparing diffusion coefficients inferred from global power balance $\left(\chi^{P B}\right)$ to those inferred from the change in the temperature gradient induced by increasing the heat flux $\left(\chi^{i n c}\right)$ or those inferred from analysis of heat pulses from sawtooth crashes $\left(\chi^{H P}\right)$. In the second section experimental data on sawtooth induced heat pulse propagation will be presented, beginning with studies of the heat pulse propagation on a fast time scale following the sawtooth crash, followed by studies of the scaling of $\chi^{H F}$. (In this paper, all heat pulses discussed are induced by sawteeth and the qualifier will be dropped for brevity.) In the third section numerical simulations of the electron temperature profile evolution following a sawtooth crash are perfurmed to investigate the relationship between the power balance studies and the heat pulse propagation studies. The simulations use either an explicit time and space dependent $\chi_{e}$, or various non-linear models for $\chi_{e}$, to provide the necessary time dependence. The experimental data are from neutral beam heated plasmas in TFTR with $I_{P} \leq 1.9 \mathrm{MA}, P_{\text {beam }} \leq 30 . \mathrm{MW}, a_{P}=0.8-0.9 \mathrm{~m}$, and $B_{\text {tor }}=4-5$ Tesla.

The original studies of heat pulses on ORMAC. ISX-B, TFTR and elsewhere relied primarily on soft $\mathrm{x}$-ray cameras, with the concomitant problems of chord averaging and lack of amplitude information. ${ }^{2.4 .14}$. Many more recent heat pulse propagation studies have used relatively fast measurements of the electron cyclotron emission from which a local electron temperature is inferred. ${ }^{5.13 .15}$ For the present studies, the principal diagnostic is the fast (200 $\mathrm{kHz}$ bandwidth) grating polychromator (GPC) which measures the electron cyclotron emission (ECE) intensity, providing a 20 point temperature profile with a channel spacing of about $6 \mathrm{~cm}$ and a radial spatial resolution of about 3 $\mathrm{cm}$. The GPC is not absolutely calibrated and thus must be cross-calibrated to another of the electron temperature profile diagnostics (i.e., the Thomson 
scattering system, the ECE radiometer or Michelson interferometer). The ability to make fast $(200 \mathrm{kHz})$, absolute measurements of the electron iemperature profile is essential for study of the heat pulse propagation in the region $r_{\text {mix }}<r<1.4 r_{m i x}$, the region where the ballistic response dominates. The use of local $T_{e}$ measurements allows direct measurement of the mixing radius for the sawtooth crash and the radial dependence of the heat pulse amplitude.

The ECE temperature diagnostics give measurements of the electron temperature profile as a function of frequency. Those frequencies must be mapped to spatial locations through a calculation of the magnetic field. For the data presented in this paper, the mapping is done using data from the SNAP code which calculates the paramagnetic, diamagnetic and poloidal field corrections to the vacuum toroidal magnetic field. The measurements are then mapped to the flux surface averaged minor radius usirg the SNAA calculation of the Shafranov shift. This mapping will not change significantly due to changes in $J(r)$ or plasma pressure during the sawtooth crash.

\section{Determination of $\chi^{\text {inc }}$}

Studies of the dependence of the total heat flux, $Q_{\text {tot }}$, on $n \Gamma T$ carried out on the JET tokamak have found that the 1-fluid incremental- $\chi, \chi_{1 f}^{\text {inc }}$, as measured from such a heat flux analysis of temperature scans is similar to the $\chi^{H P}$ (the 1-fluid $\chi_{1 f}^{\text {inc }}$ is an effective diffusivity where the heat flow through the ion and electron channels is not separated). This result suggested a common origin for the observed non-linear (offset linear) dependence of the heat flux on $n_{e} \nabla T_{e}$ and the fast heat pulse propagation (where $\nabla T=\partial T / \partial r$ ). A model was proposed for which $\chi^{H P}=\chi_{e}^{i \pi c} \geq \chi_{e}^{P B}$, and it was claimed that in general, $\chi^{H P}$ should equal $\chi^{\text {inc }}$. However, the $\chi^{H P}$ should be compared to $\chi_{e}^{P B}$ or $\chi_{e}^{i n c}$, not $\chi_{1 f}^{i n c}$. For example, the $\chi_{1 f}^{P B}$ could deviate systematically from the $\chi_{e}^{P B}$ due to systematic changes in the electron-ion heat transfer, resulting in large differences between the inferred $\chi_{e}^{i n c}$ and $\chi_{1 f}^{i n c}$. In this section we will show with data from TFTR that the experimentally determined incremental electron thermal diffusivity, $\chi_{e}^{\text {inc }}$, is not equal to the experimental $\chi^{H P}$. We will further demonstrate theoretically that only under the condition that $Q_{e}$ depends only on $\nabla T_{e}\left(\right.$ and not $\left.T_{e}\right)$, does $\chi^{H P}=\chi^{i n c}$.

A measure of the incremental electron thermal diffusivity was obtained 
on TFTR by making a scan of the electron temperature at constant electron density. This was accomplished by increasing the beam power and decreasing the gas feed to compensate for the increased fueling from the beams. A power balance analysis of the data from these scans was done using the SNAP time-independent analysis code where the power flow through the electron and ion channels was calculated separately. Measurements of the electron temperature, ion temperature, and electron density profiles were used in the analysis.

In Fig. 1 the total heat flux through the electrons for the different shots in this scan is graphed against the local $n_{e} \nabla T_{e}$ for three minor radii. Included in this scan is a shot at somewhat higher beam power for which extensive heat pulse analysis will be performed. Also included for completeness (the open symbols) is the data from an ohmic shot at the same density, but for which ion temperature profiles could not be measured. For these shots the power balance transport analysis code assumes $\chi_{i}=\chi_{e}$ and performs a series of iterations to determine the ion temperature. The points representing the beam heated data (solid symbols) show a non-linear dependence of $Q_{e}$ on either $T_{e}$ or $\Gamma T_{e}$, however, it is difficult to distinguish experimentally between an offset linear fit to the datr (if the ohmic points are eliminated) implying a model with a fixed incremental $\chi$, or a parabolic fit implying $\chi_{e} \propto T_{e}$ or $\Gamma T_{e}$. However, for the highest power point, the $\chi_{e}^{\text {inc }}$ is approximately twice $\chi_{e}^{P B}$ at all radii. It has been previously shown that $\chi^{H P} \approx 5 \chi^{P B}$ for this point. ${ }^{6}$ These results are not consistent with an incremental $\chi$ model. ${ }^{5}$ While a more general treatment of the dependence of thermal diffusivity on local parameters can reconcile the observation that $\chi^{H P} \approx 2.5 \chi^{\text {inc }}$ as will be demonstrated in the remainder of this section. a more detailed study of the heat pulse shapes in the following sections strongly suggests that the bulk of the sawtor)th induced heat pulse propagation anomaly results from the physics of the sawtooth crash itself.

The above results demonstrate that experimentally $\chi^{H P}$, which applies to small perturbations about the equilibrium profile, and $\chi^{\text {inc }}$ which applies to incremental changes in the equilibrium profile may be very different. The distinction is also necessary when considering non-linear models for $\chi_{e}$ which have a dependence on $T_{e}$ (or the temperature gradient scale length $\left.L_{T} \equiv T_{e} / \nabla T_{e}\right)$ as well as $\nabla T_{e} \cdot{ }^{16}$ The effect of this 'scale length' term in equilibrium studies is negligible; in equilibrium the shape of the temperature 
profile does not change significantly, ${ }^{7-10}$ i.e., as $T_{e} \propto \nabla T_{e}$. Conversely, in perturbation studies the relative change in $T_{e}$ is much less than the change in $\Gamma T_{e}$, making the dependence on the temperature gradient scale length important and the dependence on $T_{e}$ unimportant. In the following discussions it will be assumed that the electron temperature profile is 'resilient'.?

An expression for the effective perturbative thermal diffusivity, $\chi^{H P}$, can be determined by linearizing the thermal transport equation with $\chi=\chi(\nabla T)$

$$
\frac{3 n}{2} \frac{\partial T}{\partial t}=\frac{1}{r} \frac{\partial}{\partial r} r n \chi(\nabla T) \frac{\partial T}{\partial r}-Q
$$

to get an equation describing the evolution of the perturbed temperature, ${ }^{4}$ $\tilde{T} \equiv T(t)-<T(t)\rangle_{\text {time }}$.

$$
\frac{3 n}{2} \frac{\partial \bar{T}}{\partial t}=\frac{1}{r} \frac{\partial}{\partial r} r n \chi^{H P} \frac{\partial \bar{T}}{\partial r}
$$

where the

$$
\chi^{H P} \equiv\left[\frac{\partial[\nabla T \chi(\nabla T) !}{\partial ! \nabla T !} \quad \nabla T=\nabla T_{\circ} .\right.
$$

Thus the effective $\chi$ describing the relaxation of perturbations to the temperature is $\chi^{H P}$. In this exercise perturbations in the radiative conling. electron-ion coupling and heating terms were neglected. These terms introduce an effective damping of the heat pulse, however the time-constant for TFTR plasmas is much longer $(\approx 100 \mathrm{msec})$ than the pulse propagation time, and thus may be ignored.

The incremental $\chi$ is determined by making small changes in the equilibrium plasma temperature and measuring the resulting change in equilibrium thermal transport. For example, transport analysis of data from $T_{e}$ scans. e.g., produced by increasing the neutral beam power at constant density. show (c.f. Fig. 1) that the heat flux is not a linear function of $n_{e} \nabla T_{e}$. An incremental (for added power input) thermal diffusivity is defined as the local slope of $Q_{e}$ versus $n_{e} \nabla T_{e}$, i.e.,

$$
\chi_{e}^{i n c} \equiv \delta\left(Q_{e}\right) / \delta_{e}\left(n \nabla T_{e}\right) .
$$

As these are studies of equilibrium plasma parameters, the decoupling of temperature and temperature gradient present in the perturbative studies is 
not present. As a manifestation of the electron temperature profile resiliency observed on TFTR and other machines, both $T_{e}$ and $\Gamma T_{e}$ are changing proportionally: a dependence of $\chi$ on $T$ will affect the transport in the same manner as a similar dependence on $\nabla T_{e}$. Thus, while the definitions of $\chi^{H P}$ and $\chi^{\text {inc }}$ appear superficially similar, they are in general very different.

Three models are considered to illustrate the inherent difference between the $\chi^{H P}$ and $\chi^{i n c}$. The first model, the incremental $\chi$ model, is the simplest and is advertised as a general linearization of non-linear transport models. The second is a model commonly used in machine design studies, the RebutLallia-Watkins (RLW) transport model. ${ }^{17}$ The third is a generic power-law model of the form, $\chi \propto T_{e}^{\alpha} L_{T}^{-\beta}$ which is introduced to illustrate in general how differences in $\chi^{H P}$ and $\chi^{\text {inc }}$ can be resolved.

The incremental $\chi$ model in its simplest form is

$$
\chi(r, t)=\chi^{i n c}\left(1-\nabla T_{\text {crit }} / \nabla T\right)
$$

where $\nabla T_{\text {crit }}$ represents the critical temperature gradient at which some, unspecified, instability begins to dominate the transport. Here it will be assumed that $\nabla T_{\text {crit }}$ and $\chi^{i n c}$ are constants; in practice, of course, both parameters can have radial dependencies and some additional parameters must be introduced to deal with regions where $\nabla T<\nabla T_{\text {crit }}$. However. outside the mixing radius for sawtooth heat pulses, this model predicts that $\chi^{H P}=\chi^{i n c} .{ }^{11}$ For the data in Fig. 1, the highest power point has $\Gamma T \approx$ $2 \Gamma T_{\text {crit }}$. thus the incremental- $\chi$ model would predict that $\chi^{H P} \approx 2 \chi^{P B}$. A factor of five is observed experimentally.

The RLW model is superficially similar to the incremental $\chi$ model, but as will be seen the differences are significant. The relevant form of the RLW model for perturbative and incremental transport studies is

$$
\chi=\chi_{a n}^{r l w}\left[1-\Gamma T_{c r t t}^{\tau l w} / \Gamma T\right] .
$$

The coefficients $\chi_{a n}^{r l w}$ and $\nabla T_{c r i t}^{\tau l w}$ are not constant but depend on local plasma parameters, ${ }^{17}$ including a dependence on the temperature gradient scale length, $L_{T}$ and the local electron lemperature. These dependences may be explicitly shown as (with the reasonable assumption that the local current density is fixed):

$$
\chi=\chi_{a n} L_{o}\left(1 / L_{T}+2 / L_{n}\right)\left[1-T_{c r i t} \nabla T_{c r i t} /(T \nabla T)\right] .
$$


The parameters $L_{o}$ and $T_{\text {crit }}$ have been introduced to keep the equations dimensionally correct. They can be thought of approximately in the following way; For perturbative studies $L_{o}^{-1}=<1 / L_{T}+2 / L_{n}>_{\text {equil }}$ and $T_{\text {crit }}=T_{\text {equil }}$ and for incremental studies the parameters are approximately as defined above using the ohmic case in the scan.

For incremental transport studies (assuming profile resiliency) the strong correlation between $T$ and $\nabla T$ means that the inverse dependence of the critical temperature gradient on $T$ may be replaced by an inverse dependence on $\nabla T$ and the RLW model has the approximate form

$$
\chi=\chi_{a n}\left[1-\left(\nabla T_{\text {crit }} / \nabla T\right)^{2}\right\}
$$

which may be compared to the incremental $\chi$ form

$$
\chi=\chi^{i n c}\left[1-\nabla T_{\text {crit }} / \nabla T\right)
$$

where the expression for $\chi^{\text {inc }}$ is exactly

$$
\chi^{i n c}=\chi_{a n}\left(1+\nabla T_{\text {crit }} / \nabla T\right) .
$$

Conversely, in perturbative heat pulse studies, the gradient term in the temperature scale length is important, but the $T$ term in the critical gradient may be ignored. On TFTR the density pulse is weaker and much slower than the heat pulse (as on $\mathrm{JET}^{18}$ ), thus the perturbations to the term involving the density gradient may be ignored.

$$
\chi^{H P} \approx \chi_{a n}\left(1+\frac{1-\nabla T_{c r i t} / \nabla T}{1+2 L_{T} / L_{n}}\right) .
$$

The RLW model, predicts that $\chi^{H P} \leq 2 \chi^{i n c}$ for $\nabla T \gg \nabla T_{\text {crit }}$ and $\chi^{H P} \approx$ $\chi^{\text {inc }} / 2$ for $\Gamma T \approx \nabla T_{\text {crit }}$. In Fig. 1, the ratio of $\Gamma T$ to $\Gamma T_{\text {crit }}$ as predicted by the RLW model varies from unity (for the ohmic case) to about 5 for the highest beam power cases. This may appear surprising as $\nabla T$ changed by only a factor of two; however, the critical temperature gradient varies inversely with $T$, and hence $\nabla T_{c r i t}$ is a factor of two lower for the high power shots than for the ohmic shot. In contrast to the incremental $\chi$ model, for $\nabla T \gg \nabla T_{\text {crit }}$, as is the case for the higher power points in Fig. 1, this model predicts $\chi^{H P} \leq 2 \chi^{P B}$ 
The two models investigated above were both inconsistent with the experimental data. Experimentally it was found that $\chi^{H P} \approx 5 \chi^{P B}$, whereas the incremental- $\chi$ model predicted $\chi^{H P}=(1.5-2) \chi^{P B}$ and the RLW model predicts $\chi^{H P} \leq 2 \chi^{P B}$. However, it was demonstrated that $\chi^{H P}$ and $\chi^{i n c}$ are not in general the same for non-linear transport models. The simplest approach to interpreting the experimentally observed difference between $\chi^{H P}$ and $\chi^{\text {inc }}$ is to consider a generic power-law model of the form.

$$
\chi \propto T_{e}^{\alpha} L_{T}^{-\beta} .
$$

For this model $\chi^{H P}=(\beta+1) \chi^{F \dot{B}}$ and $\chi^{\text {inc }}=(\alpha+1) \chi^{P B}$. Thus, the experimental data for the high temperature shot in Fig. 1 might be explained with $\alpha=1$ and $\beta=4$. This implies a very strong, perhaps unrealistic, non-linear dependence of the thermal diffusivity on the temperature gradient over a wide range in temperature gradients. Below, it will be shown that an even stronger non-linearity is required to duplicate the heat pulse shape, rather than just the time-to-peak, thus it seems very unlikely that simple non-linear transport models are responsible for the rapid heat pulse propagation observed on TFTR.

\section{Experimental Heat Pulse Data}

Heat pulse propagation studies on TFTR have been done in both ohmic and neutral beam heated plasmas. A large variation in the $\chi^{H P}$ has been observed; generally on TFTR the heat pulses propagate slower in the higher density or colder plasmas. While there is a tendency for $\chi^{H P}$ to increase and 'saturate' with higher beam power, there is still significant variation of $\chi^{H P}$ at beam powers up to $5 \times P_{O H}$.

In Fig. 2 is shown the $\chi^{H P}$ (determined from simple time-to-peak analysis) for the temperature scan data shown in Fig. 1. The incremental $\chi$ model predicts that the heat pulse propagation should be invariant over this range of heating power. Indeed, for powers above about $6 \mathrm{MW}$, the $\chi^{H P}$ is very similar for all shots. However, for the lower power (coldest) shots, the heat pulse propagation is somewhat slower. In Fig. 3a, the electron temperature profiles from before and $300 \mu \mathrm{s}$ after the sawtooth crash are shown for a high and low temperature beam heated plasma with a density of $\left\langle n_{e}\right\rangle=3.8 \times 10^{19} / \mathrm{m}^{3}$. As can be seen the profile shapes, the inversion radii and the percent drop 
in the central $T_{e}$ are very similar for these shots. However, the heat pulses in the colder plasma propagated more slowly (Fig. 3b). These observations are not consistent with the incremental- $\chi$ model or other offset linear forms of non-linear transport models since even for the highest power shot shown here, $\left(P_{\text {beam }}=19 M W\right) \chi^{H P}>5 \chi_{e}^{P B}$ or $2.5 \chi_{e}^{\text {inc }}$. They could, however, be qualitatively consistent with generalized non-linear models. This result will be discussed in mere detail below.

The prediction of the RLW model regarding the scaling of $\chi^{H P}$ with density was checked by inc:easing the density at constant electron temperature. The gas feed was increased with increasing beam power so as to keep $T_{e}$ roughly constant (Fig. 4a). The low densit" shot had $\left\langle n_{e}\right\rangle=4 \times 10^{13} / \mathrm{m}^{3}$ which was increased to $\left\langle n_{e}\right\rangle=6 \times 10^{19} / \mathrm{m}^{3}$. The higher density shot had a slower heat pulse propagation by roughly a factor of five (Fig. 4b) while the $\chi^{P B}$ remained nearly constant. The $\chi^{H P}$ was calculated by the extendedtime-to-peak method ${ }^{5}$ over the radial range $0.28 \mathrm{~m}<r<0.47 \mathrm{~m}$. With this meth $\mathrm{d} \chi^{H P}=L_{\text {amp }} H_{H P}$ where $L_{\text {amp }}$ is the gradient scale length of the heat pulse peak amplitude and $V_{H P}$ is the velority of the heat pulse peaks. The RIII model predicts a weak dependence of $\chi^{H P}$ on density as $\Gamma T_{c r i t} \times n_{e}^{1 / 2}$. For this density scan the predicted changed in $\chi^{H P}$ is about $10 \%$.

Heat pulse propagation is also found to vary in ohmic plasmas; however interpretation of the results is much more difficult. Separation of the heat flow in the electron and ion channels is not feasible and it is not possible to independently vary the electron density and temperature. The data from two shots at the extremes of an ohmic density scan are shown in figure $5 \mathrm{a}$. For densities below about $<n_{e}>\approx 1.6 \times 10^{19} / \mathrm{m}^{2}$ the heat pulses were ballistic (see next section). At the highest densities in this scan, $\left\langle n_{e}\right\rangle \approx$ $3.1 \times 10^{19} / \mathrm{m}^{3}$ the heat pulses were slower than at the lower densities, but still too fast to be consistent with $\chi^{P B}$ (Fig. 5b).

\section{A. The Ballistic Response}

In the preceding section it is clear that the heat pulse propagation is faster than might be explicable by the types of transport models described in Sec. I. That, however. is not the only problem with simple interpretations of the experimental heat pulses. A careful study of the shape of the heat pulses over the radial range $r_{m i x}<r<1.5 r_{m i x}$ shows $^{6}$ that the shape of the 
heat pulses is not consistent with a simple diffusive process, i.e., $\chi_{e}$ constant in time. The initial rate-of-rise of the heat pulse is much too fast, a feature described as the 'ballistic' effect.

On TFTR the sawteeth appear to be Kadomtser-like ${ }^{19,20}$ in that there is no observed direct deposition of heat beyond the mixing radius during the sawtooth crash (however on a timescale of hundreds of $\mu \mathrm{sec}$ after the sawtooth crash, heat is transported in a diffusive manner well beyond the sawtooth mixing radius). In Fig. 6 the electron temperature profiles before and after a typical sawtooth crash are shown. The sawtooth crash results in a sharp shoulder on the temperature profile at the mixing radius of about $0.29 \mathrm{~m}$ and in a hollow temperature profile in the core. This hollow profile is more consistent with reconnection-like models than with, for example, fully stochastic models of the sawtooth crash. However, measurements of the $q$ profile find that $q(0)<1 .^{21.22}$ which casts serious doubts on reconnection models for the sawtooth crash. However, this observation makes the assumption that heat redeposition is confined to within $r_{m i x}$ at the sawtooth crash even less defensible.

Studies of heat pulse propagation on TFTR have been greatly improved over previous studies by the introduction of a GPC which can make fast, $4 \mu \mathrm{sec}$ measurements of the electron temperature profile. With this diagnostic it is possible to identify the location of the mixing radius, as well as make absolute measurements of the heat pulse amplitude versus minor radius. This diagnostic makes possible the quantitative measurement of the 'ballistic' component of sawtooth induced heat pulse propagation. This ballistic effect appears to be correlated with the strength of the $m=1$ precursor eigenmode beyond the mixing radius. ${ }^{6,23}$

In Fig. 7 the experimental heat pulses from the sawtooth in Fig. 6 are compared to simulated heat pulses using $\chi^{P B}(r)$. As well as propagating faster than the simulated heat pulses, the experimental heat pulses have a larger amplitude and a slower decay of amplitude with increased minor radius. In Fig. 8 is shown data taken at $100 \mathrm{kHz}$ during the sawtooth crash. Both the inboard and outboard electron temperature data from radii between. $r_{m i x}$ and $1.4 r_{m i x}$ are shown. At the time of the crash (approximately $0.4 \mathrm{msec}$ in the figure) a spike in the temperature lasting $20-30 \mu \mathrm{sec}$ is observable on the outboard channels $(0.31$ and $0.37 \mathrm{~m})$; this temperature rise is not due to cross-field transport, but reflects the displacement of the flux surfaces 
by the $(\mathrm{m}, \mathrm{n})=(1,1)$ precursor. Following the spike is a slower, axisymetric temperature rise over a period of $100-300 \mu \mathrm{sec}$. This is the ballistic response. The experimental data are compared to a sinulation predicting what should have been observed had the heat pulses evolved with the power balance estimate for the electron thermal diffusivity, $\chi^{P B}$. For example, at a minor radius of $0.42 \mathrm{~m}$, the electron temperature increases by $\approx 170 \mathrm{eV}$ in the $500 \mu \mathrm{sec}$ following the crash; in the simulation using $\chi^{P B}$ the change is less than $1 \mathrm{eV}$ over the same interval, a factor of 200 too small. It will be shown below that this rate of temperature rise is also much larger than would be consistent with the $\chi^{H P}\left[\equiv\left(r^{2}-r_{\text {mix }}^{2}\right) / 9 t_{\text {peak }}\right.$; or $\chi^{i n c}\left(\equiv \partial Q_{e} / \partial(\nabla T)\right)$ inferred for these plasmas.

\section{Parameter Dependence of the Ballistic Effect}

Determination of the strength of the ballistic effect is difficult as even the most strongly ballistic cases appear, at least superficially, to be 'diffusive' when viewed with relatively slow $(<5 \mathrm{kHz})$ diagnostics. Only by careful comparison of the experimental data to heat pulses simulated with rarious linear and nonlinear models is it possible to show clearly that the heat pulses have a ballistic component which cannot be modeled with a pure diffusion code. Likewise, the lack of fast temperature profile information introduces uncertainty in determining the location of the mixing radius, e.g., looking at the rate-of-rise of the heat pulse with a relatively slow. $5 \mathrm{kHz}$, diagnostic ${ }^{24.25}$ to determine the mixing radius will result in an incorrect answer. Any uncertainty in the location of the 'mixing radius' introduces further difficulties in quantifying the strength of the ballistic effect.

The origin of the ballistic response is not known at this time, however recent works by several authors ${ }^{6,23,26,27}$ have suggested a connection between the strength of the $m=1$ eigenmode beyond the $q=1$ surface and the amount of stochastic enhancement of the heat pulse propagation. With fast $(>100 \mathrm{kHz})$ measurements of the electron temperature profile evolution in plasmas rotating fast relative to the sawtooth crash times it is possible to directly measure the poloidal structure of the $n=1$ sawtooth precursor. ${ }^{20}$ $\mathrm{By}$ examination of the radial displacement of contours of constant electron temperature, it is possible to infer the displacement in the flux surface, $\xi(r)$, resulting from the $m=1$ precursor to the sawtooth crash. In Fig. 9 the 
temperature profiles before and $\approx 300 \mu$ sec after a sawtooth crash are shown. In Fig. 10 the inferred displacement of the flux surfaces on the low field side due to the mode is shown. As opposed to the 'tophat' eigenmode structure expected in cylindrical low $\beta$ models, this precursor extends well beyond the $q=1$ surface to nearly $r=0.4 \mathrm{~m}$. A simulation using the experimental heat pulse at the minor radius $r=0.28 \mathrm{~m}$ as the inner boundary condition is shown in Fig. 11a. The multiplier of 15 on $\chi^{P B}$ is chosen to match the timeto-peak at $0.56 \mathrm{~m}$. The rate of rise and amplitude of the simulated heat pulses is much smaller than experimentally observed for the channels further out. The situation is similar if the experimental heat pulse at $r=0.35 \mathrm{~m}$ is used as a boundary condition. However, using the data from $r=0.42 m$, as shown in Fig. $11 \mathrm{~b}$, it is possible to find good agreement, in this case using an effective $\chi_{e}=10 \chi^{P B}$. Thus, beyond the radial region most strongly affected by the precursor activity, the heat pulse could be considered roughly diffusive.

This result is suggestive that magnetic activity induced by the sawtooth crash does play a role in the subsequent heat pulse propagation. However. extension of the eigenmode beyond the $q=1$ surface does not guarantee that there will be stochasticity in that region. Further, such measurements of $\xi(r)$ are difficult to make. typically only being possible in beam heated plasmas which rotate relatively fast.

\section{Heat Pulse Simulations}

In this section numerical simulations based on models for the heat transport will be compared to the experimental data in order to gain deeper insight into the nature of the heat pulse propagation. The variety of models purporting to describe thermal transport in tokamaks is large; by necessity we will restrict this study to several representative models which we judge to be most relevant. We will first investigate the possibility that the thermal diffusivity is transiently affected by the passage of the heat pulse, most probably through the increase in the electron temperature gradient $\left(\nabla T_{e}\right)$. The dependence of $\chi_{e}$ on the temperature gradient can be arbitrarily complex, and we will only try two specific models, the incremental- $\chi$ model and a generic power law model. We will show that the experimental sawtooth induced heat pulse propagation is inconsistent with the $\chi^{\text {inc }}$ determined from the power scan studies. We will further show that the shape of the heat pulses cannot 
be reproduced with any reasonable model which relies only upon an implicit $\Gamma T_{e}$ dependence in $\chi_{e}$. We will then look for an explicit time-dependent form for $\chi_{e}$, beginning with an analytic expression suggested in an earlier paper by Fredrickson, et al. ${ }^{6}$ which gave qualitative agreement with the ballistic effect at $r \approx 1.4 r_{\operatorname{mix}}$ and the subsequent heat pulse propagation out to $r \approx 2.0 r_{\text {mix }}$. By adjusting the parameters in the expression it is possible to find a quantitatively correct simulation of both the ballistic response between $r=r_{\operatorname{mix}}$ and $r \approx 1.4 r_{\operatorname{mix}}$ as well as the heat pulse in the outer region. While a good fit to the experimental heat pulses may be found in this way, this approach does not address the physical mechanism responsible for the enhancement directly, as has been discussed previously. ${ }^{6}$ The first two models apply in general to transient perturbations of the temperature profile, the last model, of course. is only relevant to studies of sawtooth induced heat pulse propagation.

The object of these simulations is to develop a self-consistent model for the thermal transport which can both describe the equilibrium plasma as well as the evolution of the sawtooth induced perturbations. The numerical simulation code solves the 1-fluid (electron) heat transport equation (Eq. 1). Approximate fits to the experimental density profile, heating profile $Q$ and equilibrium $\chi$ profile are found using analytic expressions. The sawteeth are simulated as an energy conserving instantaneous reconnection. The mixing radius as well as the hollowness of the post sawtooth profile are chosen to best match the experimental data. The sawtooth crash in the simulation does not transport heat beyond the mixing radius. The radial grid in the code has a $1 \mathrm{~cm}$ spatial resolution and a Dirichlet $(\partial T / \partial t=0)$ boundary condition is enforced at $r=1 \mathrm{~m}$. The typical TFTR plasma has a minor radius of $0.8 \mathrm{~m}$; thus the code allows finite temperature and heat pulse amplitude at the plasma 'edge'. ${ }^{4}$ This approach results in smaller reflections from the plasma boundary in these simulations than would the condition that $\bar{T} / T=0$ at the edge of the plasma. However, the actual boundary condition has not been measured experimentally for these shots (due primarily to a low signal-to-noise at the plasma edge for ECE temperature measurements). The modifications to the above formula necessary to simulate the various models will be discussed below.

An important input to the simulation code is the proper reconnection or mixing radius, $r_{m i x}$. The use of a mixing radius larger than the experimental 
one will increase the heat pulse amplitude and shorten the time-to-peak, thus at larger minor radius the effect will be similar to that due to the ballistic response. Experimentally determination of the location of the mixing radius is simple with fast temperature profile data. The temperature profile is flat or slightly hollow within the mixing radius following the saw tooth crash and the mixing radius is defined as the radius at which the sharp 'shoulder' appears on the temperature profile (c.f. Fig. 6). This definition of the mixing layer is basically similar to that introduced by Sips and Lopes Cardozo where the mixing radius is defined as 'the radius out to which there is an immediate response of $T_{e}$ to the crash'. ${ }^{24}$ However, as can be seen from the data shown in Fig. 8, a digitizing rate of at least $100 \mathrm{kHz}$, not the $5 \mathrm{kHz}$ typically used by Sips et al., is necessary to properly measure the mixing radius.

The sawtooth repetition time in the experiment was about $250 \mathrm{msec}$; however the reheat had saturated at about $100 \mathrm{msec}$, so to reduce computation time, a sawtooth period of $100 \mathrm{msec}$ was used in the simulations. A few simulations were done with a $200 \mathrm{msec}$ sawtooth period, and as expected there was no effect on the heat pulse propagation. Experimentally; the reconnection was observed to leave the temperature profile temporarily hollow within the mixing radius. and a parameter to simulate this hollowness was included in the simulation code.

In addition to the above simulations. various 'perturbative' simulations using the linearized transport equations are useful. For the investigation of the incremental- $\chi$ model both an initial value, perturbative simulation and a driven boundary condition perturbative simulation (using experimental data) were performed. These simulations will be discussed in more detail below.

\section{A. Non-linear $\times$ Models}

The non-linear $\chi_{e}$ models are motivated in part by the observation that an implicit $\nabla T$ dependence in $\chi_{e}$ would result in a difference in the effective thermal transport coefficients for equilibrium versus transient heat flows. This is consistent with, but not proven by, the approximate offset linear scaling of the (one fluid) heat flux with $n \nabla T$ reported on JET and is also suggested by the many observations of profile consistency.$^{7-10}$ However, as was demonstrated above, it is very difficult to distinguish between the various possible non-linear scalings of the electron heat flux based upon power scan 
analyses and the relationship between $\chi^{H P}$ and $\chi^{\text {inc }}$ depends on the transport model assumed.

There is both experimental and theoretical support for the suggestion that $\chi=f(\nabla T)$ (c.f. Fig. 1). There are theoretical models which predict a temperature gradient dependence in the electron thermal transport, e.g., $\eta_{e}$ models ${ }^{28}$ and semi-empirical models such as the RLW model. ${ }^{17}$ In the $\eta_{e}$ models, that dependence is weak, predicting roughly a dependence of $\chi_{e}$ on the temperature gradient to the power of 1-1.5. Experimental studies of the equilibrium dependence of $\chi_{e}$ on electron temperature suggest a weak scaling, with $\chi_{e} \propto T_{e}^{0-1}$ (e.g., Fig. 1). In equilibrium, the temperature and temperature gradient are very strongly correlated, and it is not possible to separate a temperature gradient dependence from a temperature dependence. The Rebut-Lallia-Watkins model is closest to a pure incremental- $\chi$ model, where the diffusivity has an offset-linear dependence on $\nabla T$ of the form

$$
\chi_{e}(r, t)=\chi^{i n c}\left(1-R \frac{\nabla T_{o}}{\nabla T}\right), \quad \nabla T>\nabla T_{0}
$$

where $R \equiv \nabla T_{\text {crit }} / \nabla T_{0}$ and $T_{0}$ is the equilibrium temperature. The ratio $R$ and $\Gamma T_{c r i t}$ are adjustable parameters used to fit the experimentally measured $\chi^{H P}$ and $\chi_{e}^{P B}$, respectively. This functional form for $\chi_{e}(\nabla T)$ has the useful mathematical property that the diffusion equation, outside the mixing radius, is exactly separable into a part describing the equilibrium heat transport with an effective $\chi_{e}^{P B}=\chi^{\text {inc }}(1-R)$ and a 'transient' part with $\chi_{e}^{H P}=\chi^{\text {inc }}$. As mentioned previously, a constraint on this model is that these equations are only valid bevond the mixing radius where the expression for $\chi_{e}$ is positive. In any region of the plasma where the temperature gradient falls below $R \nabla T_{o}$ this model predicts a negative value for $\chi_{e}$.

Sips et al. have attempted to simulate the heat pulse data shown in Fig. $T$ using an incremental- $x$ model of the form $\chi^{\text {inc }}=1.0+14 \sqrt{r / a}$. In their initial value simulations they found it necessary to use a mixing radius of $r_{m i x}=0.34 \mathrm{~m}^{24}$ From the fast temperature profile data, however, the mixing radius can be determined quite accurately as $r_{\text {mix }}=0.29 \pm 0.01 \mathrm{~m}$ (Fig. 12). With the proper mixing radius and this model for $\chi^{\text {inc }}$ the amplitude of the heat pulse is too small and the rate-of-rise is too slow at the minor radius of about $0.4 \mathrm{~m}$. Thus, by using a mixing radius $20 \%$ larger, $r_{\text {mix }}=0.34 \mathrm{~m}$, than the true mixing radius, $r_{\text {mix }}=0.29 \mathrm{~m}$, Sips and Lopes 
Cardozo were able to approximately simulate the effects of the ballistic response for $r>1.4 r_{\text {mix }},{ }^{24.25}$ i.e., outside the region of the strong ballistic response. If the ballistic effect is properly simulated within $1.4 r_{\text {mix }}$, it is possible to approximately simulate the heat pulses in the region beyond $r \approx 1.4 r_{m i x}$ with a number of diffusive and non-diffusive models.

The simulation described above by Sips and Lopes Cardozo was a linearized initial value simulation or perturbative simulation. For a non-linear simulation of heat pulse propagation with this model, it is necessary to deal gracefully with the constraint that $\chi_{e}>0$. In previous perturbative simulations, the approach has been to use a very low value of $\chi_{e}$ within the mixing radius. In the same spirit, the non-linear simulations were done with the mudel

$$
\chi(r, t)=0.001+\chi^{P B}(r)\left(\Gamma T / \Gamma T_{0}\right)^{4} \quad \nabla T<\nabla T_{0} .
$$

The actual $\chi_{e}(\nabla T)$ used is shown in Fig. 13. In the same figure, for comparison, is the $\chi_{e} v s . \Gamma T$ for the other two models discussed below.

Simulations using the $\chi^{\text {inc }}$ determined from the temperature scans at constant density discussed above show that the heat pulses propagate approximately a factor of two slower than the experimental data. To match the time-to-peak of the experimental data, it is necessary to use $\chi_{e}^{H P}=$ $2.5 \chi^{i n c}=5 \chi_{e}^{P B}$, i.e.. $R=0.8$. In Fig. 14 the heat pulses simulated with this model are compared to the experimental heat pulses. While the time-topeak and general shape of the heat pulses agree fairly well, the heat pulses are about a factor of two too small. The conclusion is that while this model does enhance $\chi$, creating a 'ballistic' effect, the enhancement in $\chi$ is too weak near the mixing radius and occurs too late at larger radii. At the minor radius of $0.42 \mathrm{~m}$. the increase is only $30 \mathrm{eV}$ in the $500 \mu \mathrm{sec}$ after the crash compared to the experimentally measured increase of $170 \mathrm{eV}$.

In the context of the simulations as done so far, there are no adjustable parameters available to improve the fit with this simulation. However, by using a stronger non-linear power-law model

$$
\chi_{e}(r, t)=\chi_{e}^{P B}\left(\nabla T / \nabla T_{0}\right)^{4}
$$

it is possible to increase the enhancement in $\chi$ near the mixing radius. (Here $\nabla T_{0}$ is the equilibrium temperature gradient.) In Fig. 15 the heat pulses simulated with this model are compared to the experimental heat pulses. At larger radii, the agreement is of similar quality to the $\chi^{\text {inc }}$ simulation of 
figure 14. However, near the mixing radius, this power law model gives a somewhat stronger 'ballistic' effect. The exponent of 4 was chosen such that if this model were linearized to the incremental- $\chi$ form, the result would be equivalent to Eq. (9). In general, linearization of power-law models of the form $\chi \propto \nabla T^{\alpha}$ to the incremental $\chi_{e}$ form gives:

$$
R=\alpha /(\alpha+1)
$$

and

$$
\chi^{i n c}=(1+\alpha) \chi_{e}^{P B} .
$$

Thus, for $R=0.8$, as used in the $\chi^{i n c}$ simulation, $\alpha=4$.

The cause of the difference between the incremental and power law models is clear; while the linearization of this model is identically the $\chi^{i n c}$ model, the linearization breaks down near the mixing radius where the change in temperature gradient can be more than a factor of two. Thus the amount of enhancement to $\chi_{e}$ is much greater with this model than with the $\chi^{\text {inc }}$ model near the mixing radius and the heat pulses come closer to matching the ballistic response. However, even with a dependence on $\nabla T$ to the fourth power this model still does not give a strong enough "ballistic" effect. Obviously a much stronger enhancement in $\chi_{e}$. i.e.. a stronger non-linearity than $(\nabla T)^{4}$ is required to match the experimentally measured heat pulse amplitude and ballistic contribution.

Rather than investigate more complicated dependencies of $\chi_{e}$ on $\nabla T$, we will use simulations using the linearized equations to determine to what extent the heat pulses are actually purely diffusive, i.e., describable by a diffusion equation (linearized) with a time-independent $\chi_{e}$. We will begin with driven boundary condition simulations. We use the experimentally measured heat pulse at a given radius as the inner boundary condition in the simulation. As before, we will use $\chi^{e f f}=5 \chi^{P B}$, or $\chi^{e f f}=2.5 \chi^{i n c}$, to match the time-to-peak.

In Fig. 16a the heat pulses are simulated using the experimental heat pulse immediately outside the mixing radius, at $r=0.31 \mathrm{~m}$. In $\chi_{\text {inc }}$ models the heat pulse propagation should be diffusive beyond that point. While the heat pulse amplitude and time-to-peak agree fairly well, there is still a significant discrepancy in the rate of rise of the heat pulse at $r=0.36 \mathrm{~m}$ (shown as the shaded region in Fig. 16a). The obvious conclusion is that the heat pulse propagation is not diffusive between $0.31 \mathrm{~m}$ and $0.36 \mathrm{~m}$. The 
simulations can be done using the experimental heat pulses at larger minor radii. In this manner it is found that only bevond $r=0.42 m$ are the heat pulses 'diffusive' (Fig. 16b) and the $\chi_{e}^{H P}$ for this model is $\approx 5 \chi_{e}^{P B}$ or $\approx 2 \chi^{\text {inc }}$. (For the RLW model $\chi^{H P} \leq 2 \chi^{P B}$ with $\nabla T=5 \Gamma T_{\text {crit. }}$ )

\section{B. Analytic Approximation to $\chi(r, t)$}

In the previous section various models with implicit transient enhancements in $\chi$ resulting from assumed non-linearities in the thermal diffusivity were investigated. The principal failure of these models was that the enhancement in $\chi$ near the mixing radius was too weak, resulting in a heat pulse amplitude which was too small. While very strong non-linearities in the heat flux might explain this ballistic effect, in the case of sawtooth induced heat pulse propagation, it is of course possible that the sawtooth crash transiently enhances the diffusivity beyond the mixing radius following the sawtooth crash. In the reconnection model of the sawtooth crash an enhancement in the thermal diffusivity is predicted in numerical studies. The enhancement is due to the effect of finite pressure and toroidicity ${ }^{23.26 .27}$ on the $m=1$ sawtooth precursor, leading to a stochastic magnetic field structure. The theoretical model is sufficiently complex that no quantitative predictions of the level of stochasticity, nor of the effect of said stochasticity on thermal transport can be made. Qualitatively, it might be expected that the stochastic regions would most strongly affect electron thermal transport and have much less of an effect on ion thermal transport or particle transport. Ion heat pulse propagation measurements have not been made, but the particle pulse does move an order of magnitude slower than the electron heat pulse in TFTR (and JET ${ }^{18}$ ). Likewise, the stochastic regions would be expected to be strongest near the mixing radius, which will be seen to be consistent with the experimental observations. Here we will use an explicit model for the temporal and spatial enhancement in $\chi_{e}$ and attempt to reproduce the heat pulse shape just outside the mixing radius. We will also demonstrate that at larger minor radii $\left(r>1.4 r_{m i x}\right)$ the shape of the heat pulse is relatively insensitive to the form of the enhancement.

A very simple analytic expression for a time dependent $\chi_{e}$ with only three adjustable parameters has been found to give a very good qualitative fit to 
the experimental data. The time dependant $\chi_{e}$ has the form

$$
\chi_{e}(r, t)=\chi_{e}^{P B}(r)\left[1-\delta \exp \left(-t / \tau-\kappa r^{2}\right)\right] .
$$

This form of the $\chi(r, t)$ gives an enhancen $e^{2}$ t in $\chi_{e}$ that is strong near the mixing radius, and falls off rapidly with larger $r$. Near the mixing radius, the enhancement is necessary for only a short time $(\approx 1 \mathrm{msec})$ following the sawtooth crash. With this model, the simulated heat pulses were found to have a rapid temperature rise, even at large minor radius and a relatively slow fall-off in amplitude with minor radius. The equilibrium temperature profile is accurately simulated as well as the sawtooth heat pulse. While this expression enhances $\chi(r, t)$ in the core. it is found that the simulations are insensitive to the enhancement in this region. The enhancement can be turned off for $r<0.8 r_{m x x}$ with no measurable effect on the heat pulse shapes bevond $r_{m i x}$. This is to be expected as the temperature gradient is very small in the core region.

In Fig. 17 the simulated and experimental temperature perturbations are compared and in Fig. 18 the temporal evolution of the perturbations are compared on a shorter timescale. On both short and long timescales, the quantitative agreement is very good. This form of the $\chi$ enhancement was chosen to best reproduce the ballistic effect, i.e., to give the best simulation of the heat pulse at $r=0.36 \mathrm{~m}$. The qualitative difference between this model and the implicit models tested above are that the perturbation to $\chi_{e}$ occurs simultaneously at all radii, and that the enhancement is much stronger near the mixing radius with this model.

As is seen in Fig. 17, this model simulates the heat pulses quite well at larger minor radius. In particular, it has been shown above that the heat pulses beyond about $r=0.42 m$ pulses can be fit with an incremental- $\chi$ model. As can be seen in Fig. $1 i$ the simulated heat pulses with this explicit model agree very well with the experimental data out to $r=0.52 m$, thus, the shape of the heat pulses in this region is not particularly sensitive to the exact form of the enhancement in $\chi$. This result is contrary to what has previously been assumed ${ }^{25}$ and calls into question the usefulness of studying the heat pulses in this region. That is, it may be possible to demonstrate that the heat pulses are consistent with a particular model, however, it is not possible to prove that only one model could explain the data. 


\section{Summary}

Simple non-linear models, such as the incremental- $\chi$ model or the Rebut-Lallia-Watkins model fail to reproduce the time behavior of the heat pulses and predict too small an amplitude of the heat pulse near the mixing radius. The heat pulses at radii greater than aboul: $1.4 r_{\operatorname{mix}}$ can be simulated with non-linear models. However, models with unacceptably strong nonlinearities are required; in the outer region, $r>1.4 r_{\text {mix }}$, models with a dependence on the temperature gradient to the fifth (and higher) power are required.

There is strong circumstantial evidence to support sawtooth induced enhancements in electron thermal transport in the ballistic region, i.e., typically between $r_{m i x}$ and $1.4 r_{m i x}$ in beam heated TFTR plasmas. There is, at present, no way to verify such a hypothesis, nor to prescribe boundaries beyond which such an effect may no longer be important. Further, because very different time-dependent forms of $\chi$ can result in qualitatively similar heat pulse shapes, there is no justification for claiming that beyond, for example, $r=1.4 r_{\text {mix }}$ the transport is diffusive.

More generally, it has been shown that the application of knowledge acquired from transient transport studies to equilibrium confinement scaling is not direct. Transient studies break constraints on plasma parameters that are present in equilibrium studies. Thus, the $\chi^{2 n c}$ determined from equilibrium temperature scans and the $\chi^{H P}$ determined from transient transport studies are only indirectly related. Other effects which have recently been invoked in the study of heat pulse propagation ${ }^{12,29,30}$ may still be important in understanding transient transport phenomena, but great care must be exercised in their application to the analysis of sawtooth-induced heat pulse propagation.

While these observations pertain to the TFTR plasmas discussed above, the possibility that sawteeth can directly affect the heat pulse propagation should be of concern in any study of sawtooth induced heat pulse propagation and possibly in any heat pulse study where sawteeth are present. While modulated electron cyclotron heating experiments present an attractive alternative to sawtoot'i induced temperature perturbations, the effects of possible sawtooth induced enhancements in the thermal transport on the effective ECH deposition must be considered. 


\section{Acknowledgments}

The authors would like to thank W. Stodiek, and J. Callen for many useful discussions and suggestions. This work was supported by US DOE Contract No. DE-AC02-76CH0-3073. 


\section{References}

${ }^{1}$ Liewer, P. C., Nucl. Fusion 25 (1985) 543.

${ }^{2}$ Callen, J. D. and Jahns, G. L., Phys. Rev. Lett. 38 (1977) 971.

33ahns, G., Soler, M., et al., Nucl. Fusion 18 (1978) 609.

${ }^{4}$ Fredrickson, E. D., Callen, J. D., McGuire, K.M., Bell, J. D., Colchin, R. J., Efthimion, P. C., Hill, K. W., Lzzo, R., Mikkelsen, D. R., Monticello, D. A., Paré, V., Taylor, G. and Zarnstorff, M., Nucl. Fusion 26 (1986) 849.

${ }^{5}$ Tubbing, B. J. D., Lopes Cardozo, N. J. and Van Der Wiel, M. J., Nucl. Fusion 27 (1987) 1843.

${ }^{6}$ Fredrickson, E. D., McGuire, K. M., Cavallo, A., Budny, R., Janos, A., Monticello, D., Nagayama, Y., Park, W., Taylor, G., and Zarnstorff, M. C., Phys. Rev. Lett. 65 (1990) 2869.

${ }^{7}$ Fredrickson, E. D., McGuire, K. M., Goldston. R. J., et al., Nucl. Fusion 27 (1987) 1897.

${ }^{8}$ Taylor, G., Fredrickson, E. D., Grek, B., Goldston, R. J., et al., Nucl. Fusion 29 (1989) 3.

${ }^{9}$ Becker, G., Nucl. Fusion 31 (1991) 663.

${ }^{10}$ Düchs, D. F., Stringer, T.E., Taroni, A., Bursati, M., Gottardi, N., Hellsten, T., Tibone, F., at 11 th Int. Conf. on Plasma Phys. and Cont. Nusl. Fusion Research, Kyoto, Japan, 13-20 Novermber 1986 Vol. I, 325.

${ }^{11}$ Lopes Cardozo, N. J., Sips, 1. C. C., Costley. A. E., Hogeweij, G. M. D., and O'Rourke, J., in Proceecings of the European Physical Society. Berlin. Vol. I, (1991) 193.

${ }^{12}$ Gentle, K. W., Phys. Fluids 31 (1988) 1105.

${ }^{13}$ Goedheer, W. J., Nucl. Fusion 26 (1986) 1043.

${ }^{14}$ Bell, J.D., Dunlap, J. L., Pare', V. K., et al. Nucl. Fusion 24 (1984) 997. 
${ }^{15}$ Riedel, K.S., Eberhagen, A., Gruber, O., Lackner, K., et al., Nucl. Fusion 28 (1988) 1509.

${ }^{16}$ Efthimion, P. c., Mansfield, D. K., Stratton, B. C., Synakowski, E., el al., Phys. Rev. Lett. 66 (1991) 421.

${ }^{17}$ Rebut, P. H., Watkins, M. L., Gambier, D. J., and Boucher, D., Phys. Fluids B 3 (1991) 2209.

${ }^{18}$ Lopes Cardozo, N. J. and de Haas, J.C.M., Nucl. Fusion 30 (1990) 521.

:9Kadomtsev, B. B., Fiz. Plazmi Vol. 1 (1975) 710; Sov. J. Plasr a Phys. $1(1975) 389$.

${ }^{20}$ Nagavama, Y., McGuire, K.M., Bitter, M., Cavallo. A. et al., Phys. Rev. Lett. 67 (1991) 3527.

${ }^{21}$ Soltwisch, H. and Stodiek, W., in Plasma Physics and Controlled Nuciear Fusion Research 1986 (Proc. 11th Int. Conf. Kyoto, 1986) (1987) Vol. I $\Omega 63$.

${ }^{22}$ Batha. S., Levinton, F., Bell, M., Kessel, C., and Weiland, R. M., in Bull. Am. Phys. Suc. 37 (1992) 1481.

${ }^{23}$ Park W., Monticello, D. A., Fredrickson, E. D., and McGuire, K. M., Phys. Fluids B 3 (1991) 507.

${ }^{24}$ Sips, A. C. C., Lopes Cardozo, N. J., Costley, A. E., Horjeweij, G. M. D.. and O'Rourke, J., in Proceedings of the European Physical Society, Berlin. vol. I. (1991) 193.

${ }^{25}$ Lopes Cardozo, N. and A. C. C. Sipps, Plasma Physics and Controlled Fusion 33 (1991) 1337.

${ }^{26}$ Aydemir, A. Y., Wiley, J.C., and Ross, D. W., Phys. Fluids B 4 (1989) 774 .

${ }^{27}$ Baty. Proceedings of the 17th European Physical Society Conf. on Controlled Fusion and Plasma Heating, Amsterdam, Vol. 14B, Part II (1990) 889. 
${ }^{28}$ Guzdar, P. N., Liu, C. S., Dong, J. Q., and Lee, Y. C., Phys. Rev. Lett. 57 (1986) 2819.

${ }^{29}$ Hossain, M., Kress, M., Hu, P. N., Blank, A. A., and Grad, H., Phys. Rev. Lett. 58 (1987) 487.

${ }^{30}$ Bishop, C. M. and Conner, J. W., Plasma Physics and Controlled Fusion, 32 (1990) 203. 


\section{List of Figures}

Figure $1 Q$ vs. $n \Gamma T$ at three radii for an electron temperature scan at constant density. The ohmic points in the scan are represented by open symbols and the points with NBI by closed circles. The temperature scan data can be equally well fit by the $\chi^{\text {inc }}$ model or a linear dependence of $\chi$ on $T$.

Figure 2 The $\chi^{H P}$ determined from time-to-peak analysis vs. the ratio of the temperature gradient to the critical temperature gradient.

Figure 3a The electron temperature profiles from before (solid line) and $\approx 300 \mu$ s after (dashed line) sawtooth crashes in beam heated plasma at similar density $(58554,58564)$. Parameters for this shot were $\left\langle n_{e}\right\rangle=3.8 \times$ $10^{18} / \mathrm{m}^{3}, I_{p}=1.8 M A, P_{N B I}=4 M W$ and $17 M W$.

Figure $3 \mathrm{~b}$ Comparison of the heat pulses for the sawteeth shown in Fig. 3a. The dashed curve is for the low temperature case.

Figure $4 \mathrm{a}$ The electron temperature profiles from before and $300 \mu \mathrm{s}$ after a sawtooth crash in $1.8 \mathrm{MA}$ beam heated plasmas (62270.62248). The density and beam power for these shots were, respectively, $\left\langle n_{e}\right\rangle=4 \times 10^{19} / \mathrm{m}^{3}$ and $6 \times 10^{19} / \mathrm{m}^{3}, P_{N B I}=7.5 \mathrm{MW}$ and $15 \mathrm{MW}$.

Figure $4 \mathrm{~b}$ Comparison of the heat pulses for the sawteeth shown in Fig. 4a. The dashed curve is for the low density case.

Figure 5a Temperature profiles before and after the sawtooth crash for a high and low density ohmic plasma $(45455,45450)$. The lower density case has the higher temperature.

Figure 5b Comparison of the heat pulses for the high density (solid line) and low density (dashed line) sawteeth shown in Fig. 5a.

Figure 6 Profiles of the electron temperature before (solid) and $200 \mu \mathrm{sec}$ after (broken) a sawtooth crash (The parameters for this shot were $I_{p}=1.7$ $\mathrm{MA}, q(a)=4.4, \mathrm{NBI}$ power $=19 \mathrm{MW}, \mathrm{a}=0.8 \mathrm{~m}, \mathrm{R}=2.45 \mathrm{~m}$ and $B_{T}=$ 4.7 Tesla.)

Figure 7 Comparison of heat pulse data from ECE emission (solid line) to simulated heat pulses (dashed line) where the power balance thermal diffusivity profile has been used, $\chi(r)=\left(0.3+9(r / a)^{2}\right) e^{\left(2.6(r / a)^{2}\right)}$.

Figure 8 The heat pulses shown in Fig. 7 , but now with faster sampling showing the ballistic response (solid line) and the simulated heat pulse (dashed line). 
Figure 9 The electron temperature profiles from before and $\approx 300 \mu$ s after a sawtooth crash in a beam heated plasma. Parameters for this shot were $\left\langle n_{e}\right\rangle=4.0 \times 10^{19} / \mathrm{m}^{3}, I_{p}=1.7 \mathrm{MA}, P_{N B I} \approx 28 \mathrm{MW}$.

Figure 10 The radial displacement of the constant temperature surfaces, $\xi(r)$ on the outboard (low field) side due to the $(m, n)=(1,1)$ sawtooth precursor.

Figure 11a Comparison of the experimental heat pulses (solid line) to simulated heat pulses (dashed line) using the experimental pulse shape at $r=0.28 m$ as the inner boundary condition.

Figure 11b Comparison of the experimental heat pulses (solid line) io simulated heat pulses (dashed line) using the experimental pulse shape at $r=0.42 m$ as the inner boundary condition.

Figure 12 Comparison of the radial profiles of the electron temperature from experiment and the simulations shown in Figs. 7,8,11 and 14 through 18 before and after the sawtooth crash as well as the profile after the crash as used by Sips and Cardozo. ${ }^{24}$

Figure 13 The normalized $\chi$ vs. the normalized $\nabla T$ for the power law model and the $\chi^{\text {inc }}$ model. Also shown is normalized $\chi$ vs. the normalized $\nabla T$ at a radius of $0.34 \mathrm{~m}$ for the simulation shown in Fig. 17.

Figure 14 Comparison of the experimental heat pulses (solid line) with a non-linear simulation (dashed line) using $\chi(r, t)=5 \chi^{P B}\left(1-.8 \Gamma T_{0} / \nabla T\right)$ for $\Gamma T>\Gamma T_{\mathrm{o}}$.

Figure 15 Comparison of the experimental heat pulses (solid line) with a non-linear simulation (dashed line) using $\chi(r, t)=\chi^{P B}(r)\left(\nabla T / \nabla T_{0}\right)^{4}$ for $\nabla T>0$.

Figure 16a Comparison of experimental (solid line) and simulated (dashed line) heat pulses where the boundary condition at $r=0.31 \mathrm{~m}$ is the experimental shape of the heat pulse and $\chi=5 \chi^{P B}$.

Figure 16b Comparison of experimental (solid line) and simulated (dashed line) heat pulses where the boundary condition at $r=0.42 m$ is the experimental shape of the heat pulse and $\chi=5 \chi^{P B}$.

Figure 17 Comparison of the experimental heat pulses from Fig. $6-8$ (solid line) with heat pulses simulated using a time dependent thermal diffusivity of the form $\chi(r, t)=\chi^{P B}(r)\left(1+35 \exp \left(-1000 t-16 r^{2}\right)\right.$ (dashed line).

Figure 18 Comparison of the experimental (solid line) and simulated 
(dashed line) fast time behaviour of the heat pulses for radii less than $1.4 r_{\text {mix }}$. 


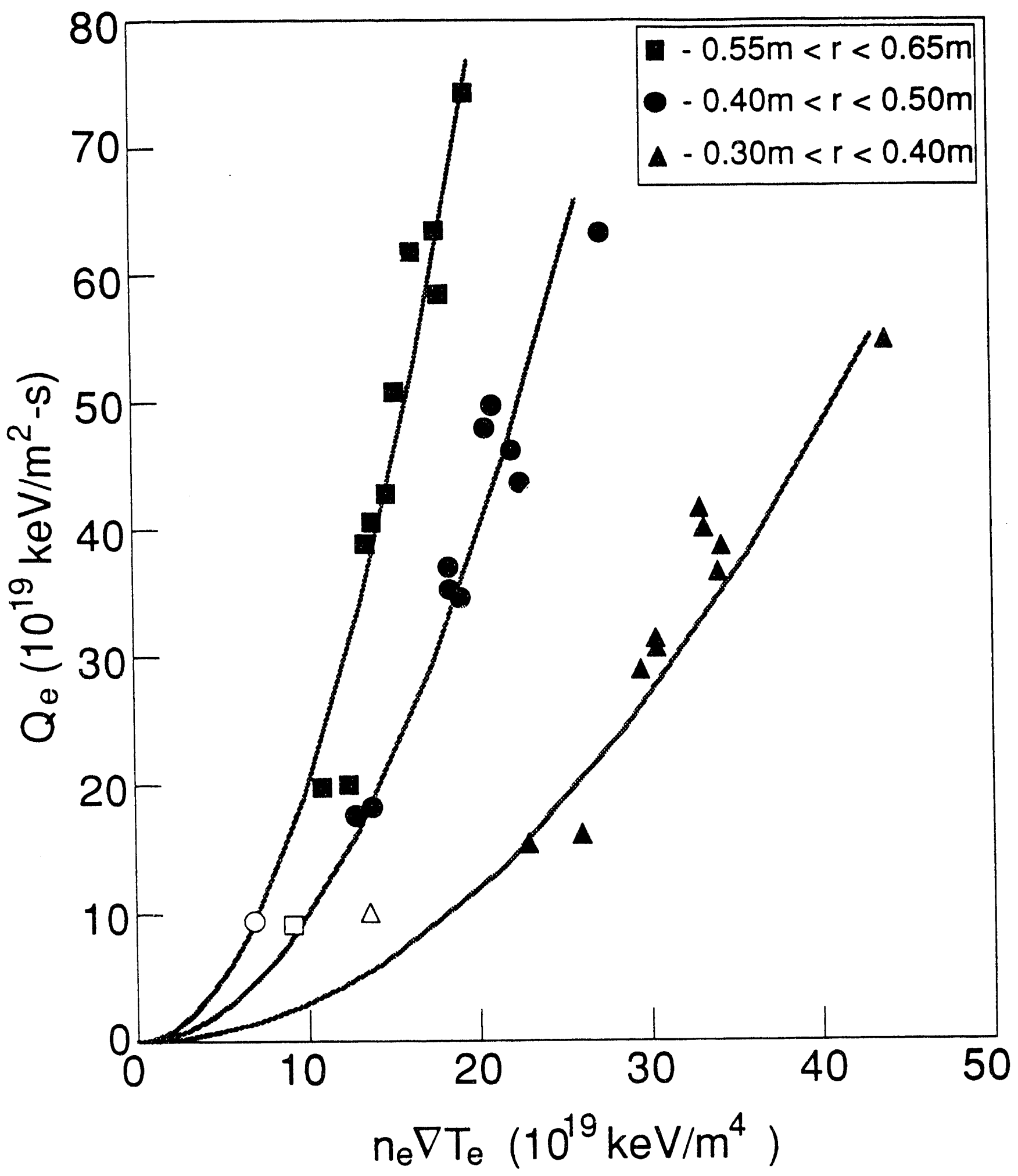

Figure 1 


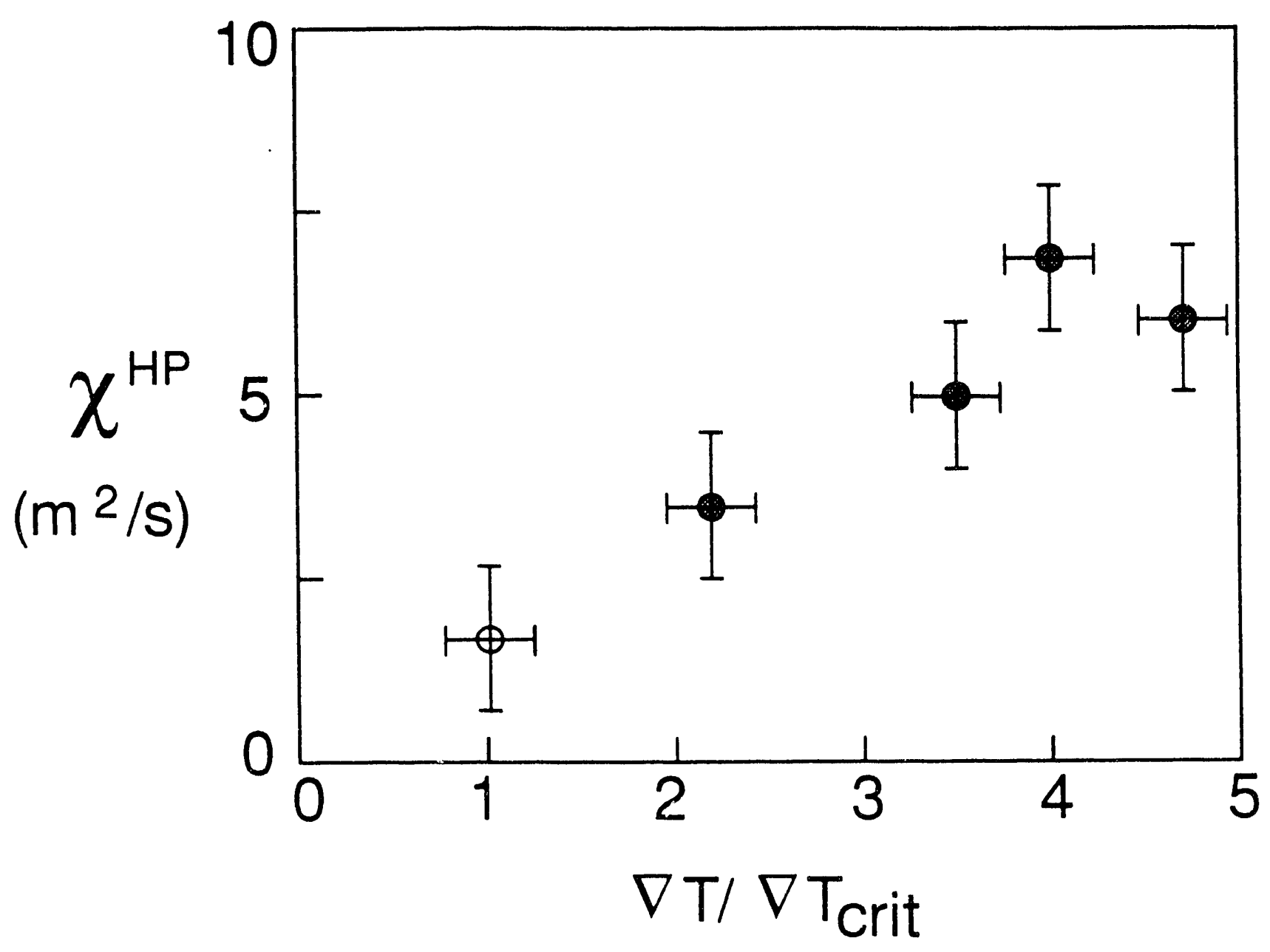

Figure 2 


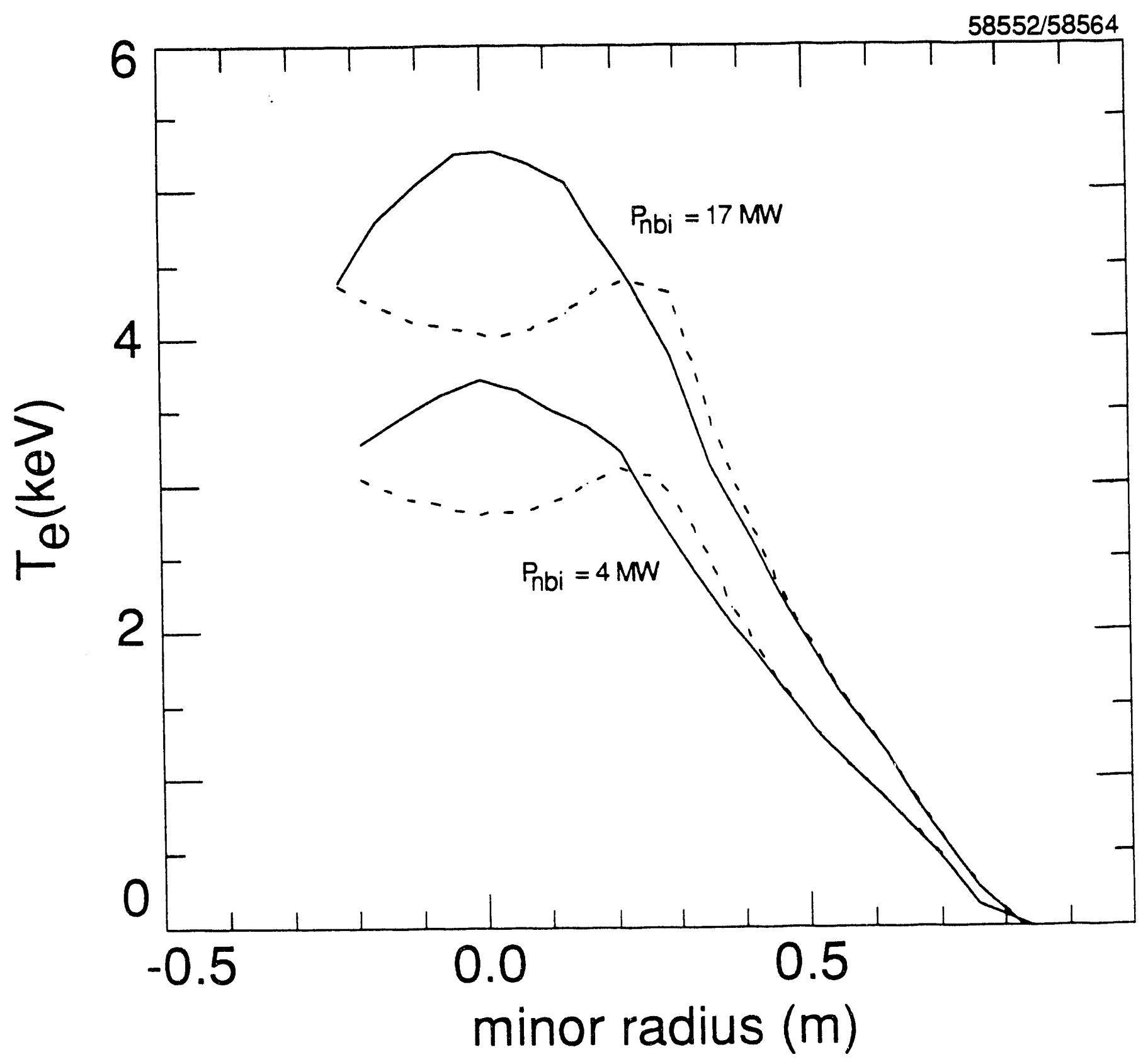

Figure $3 a$ 


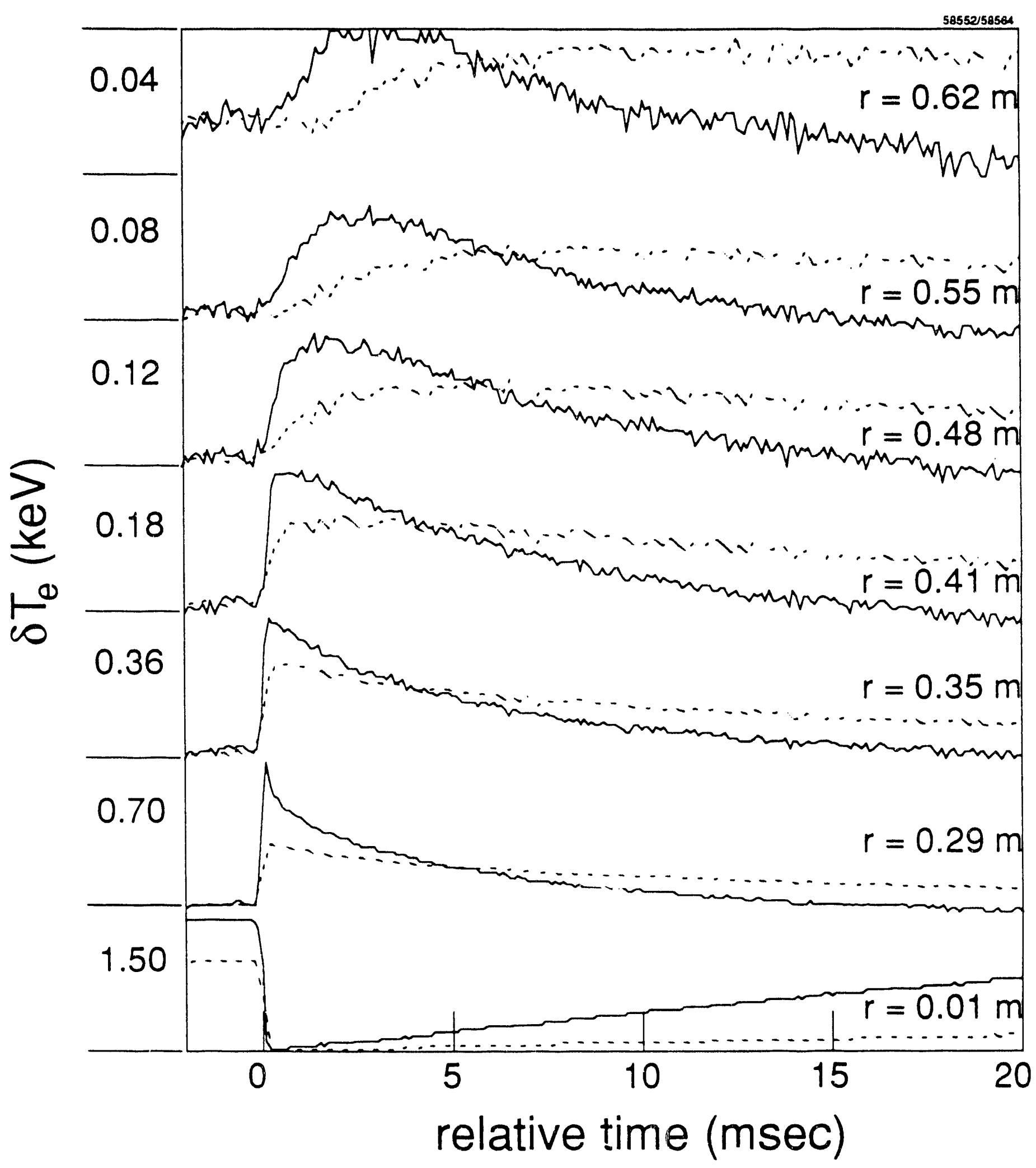

Figure $3 b$ 


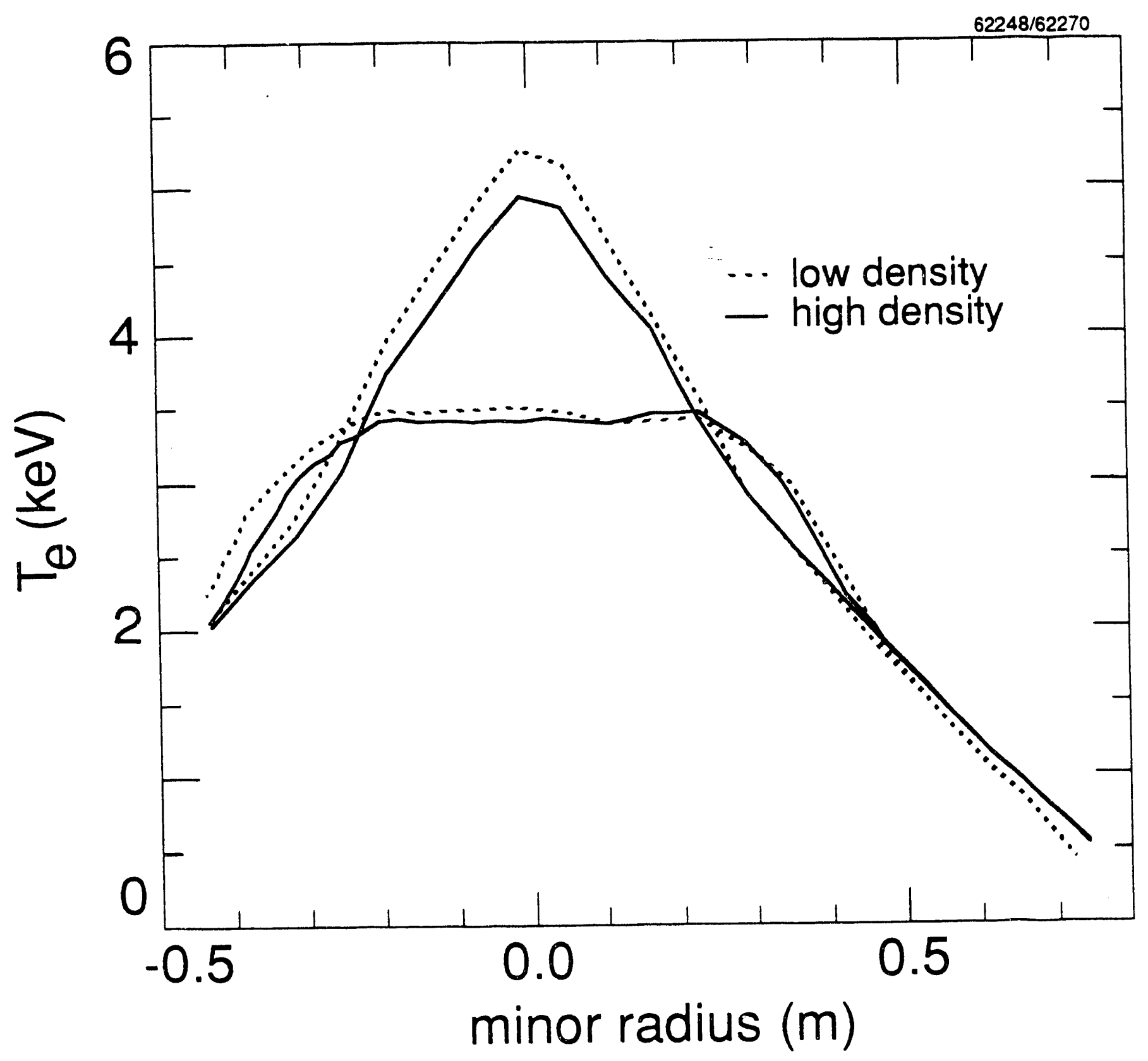

Figure 4a 


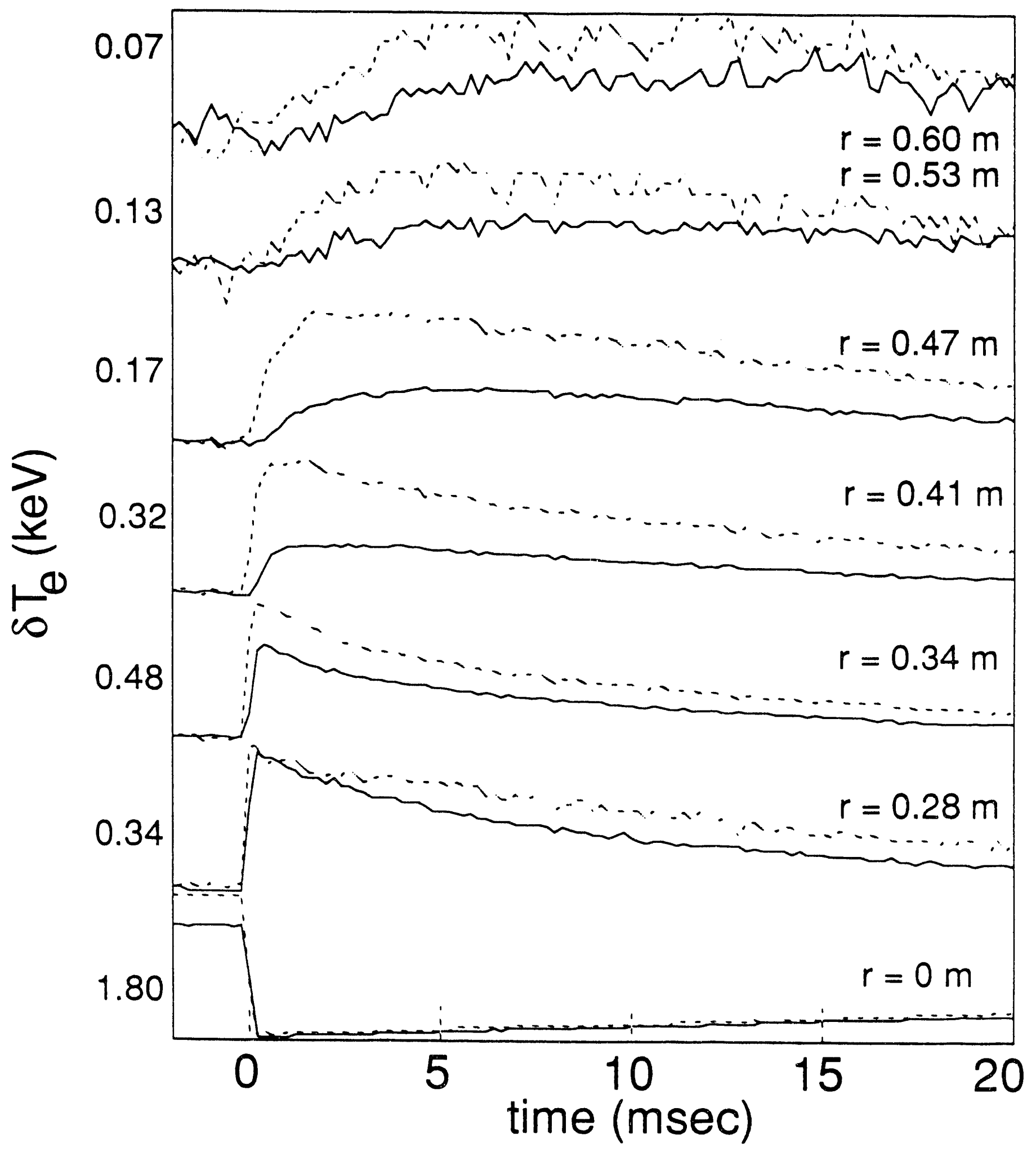

Figure $4 b$ 


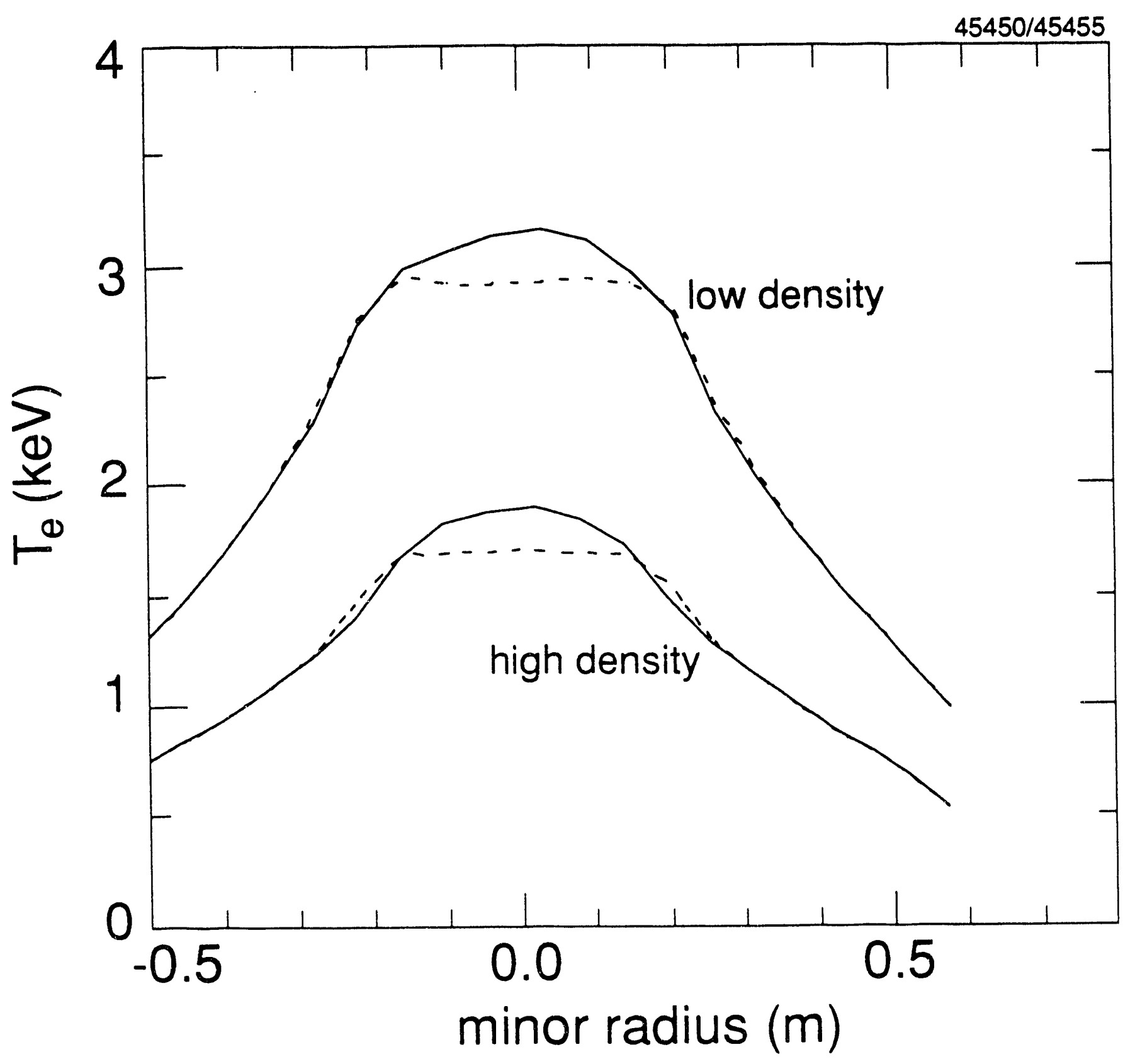




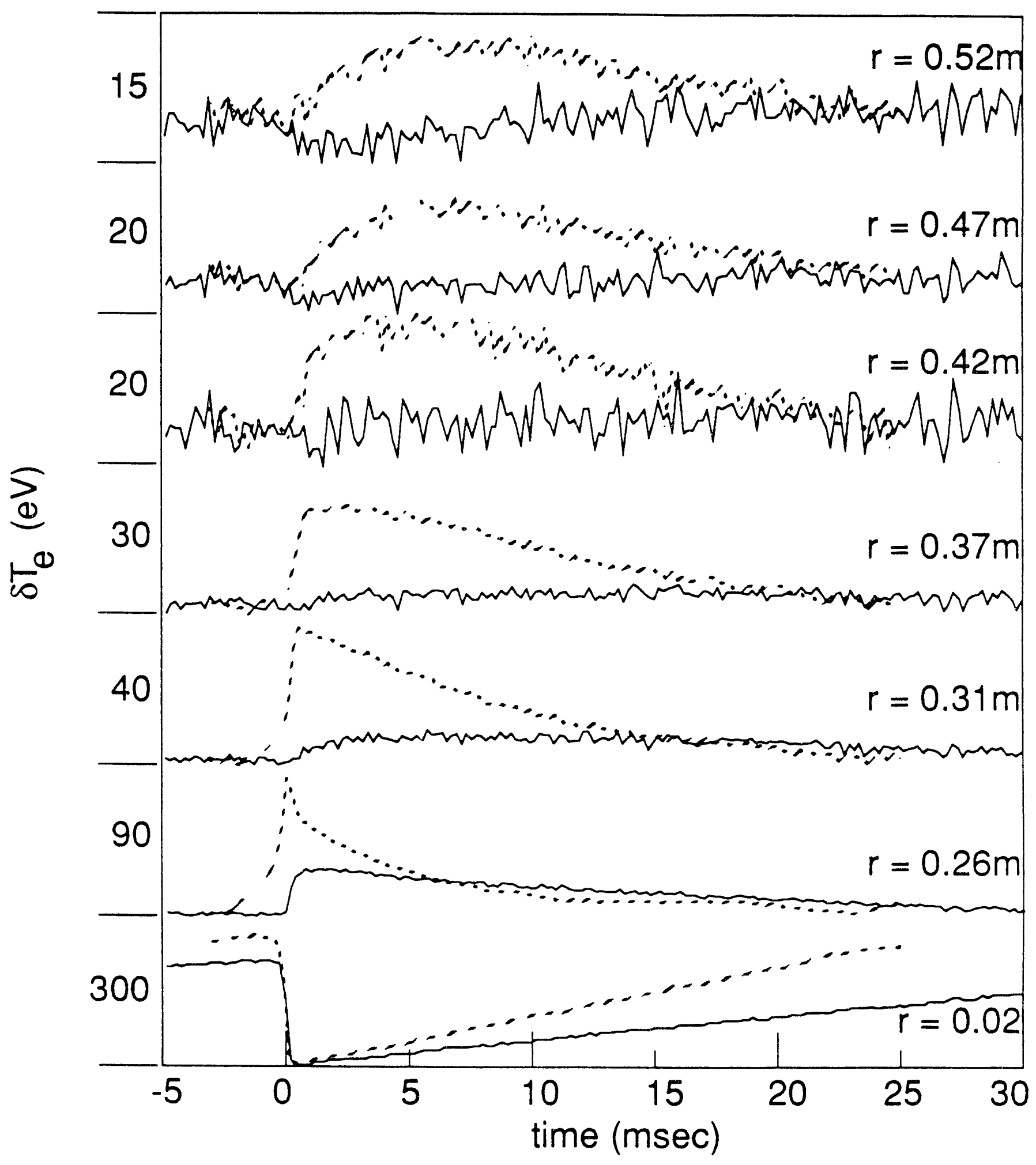

Figure 5b 


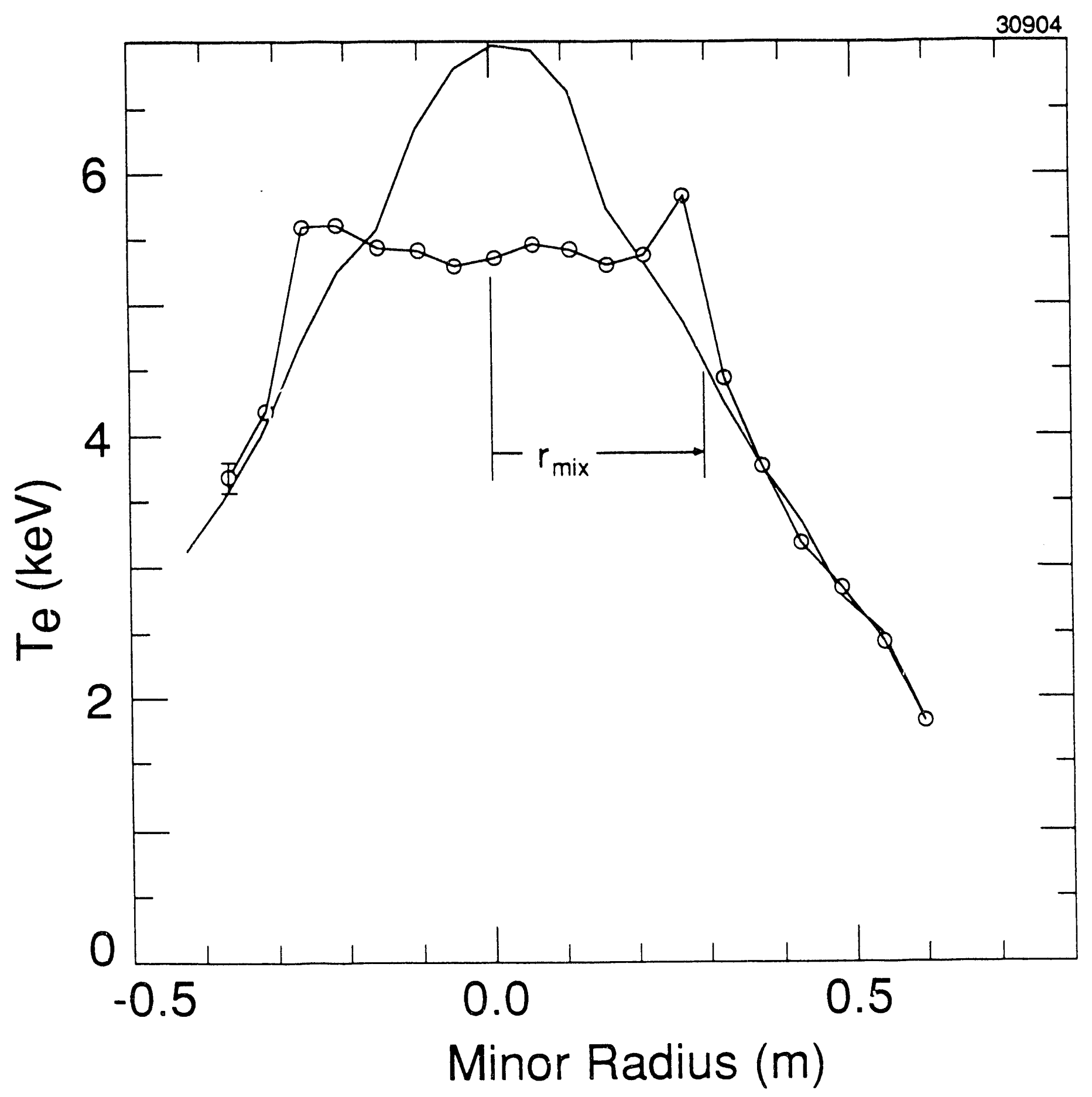

Figure 6 


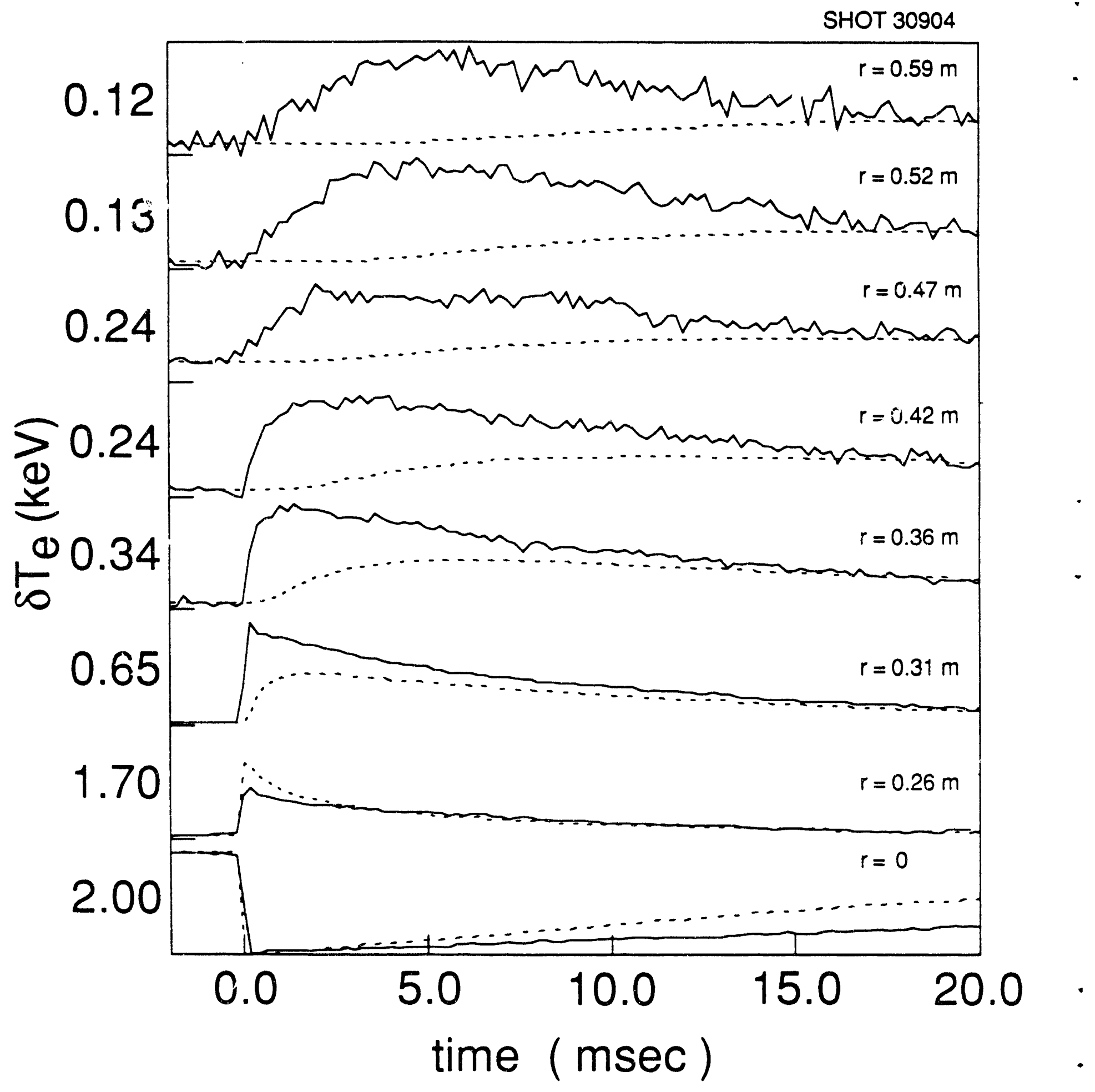

Figure 7 


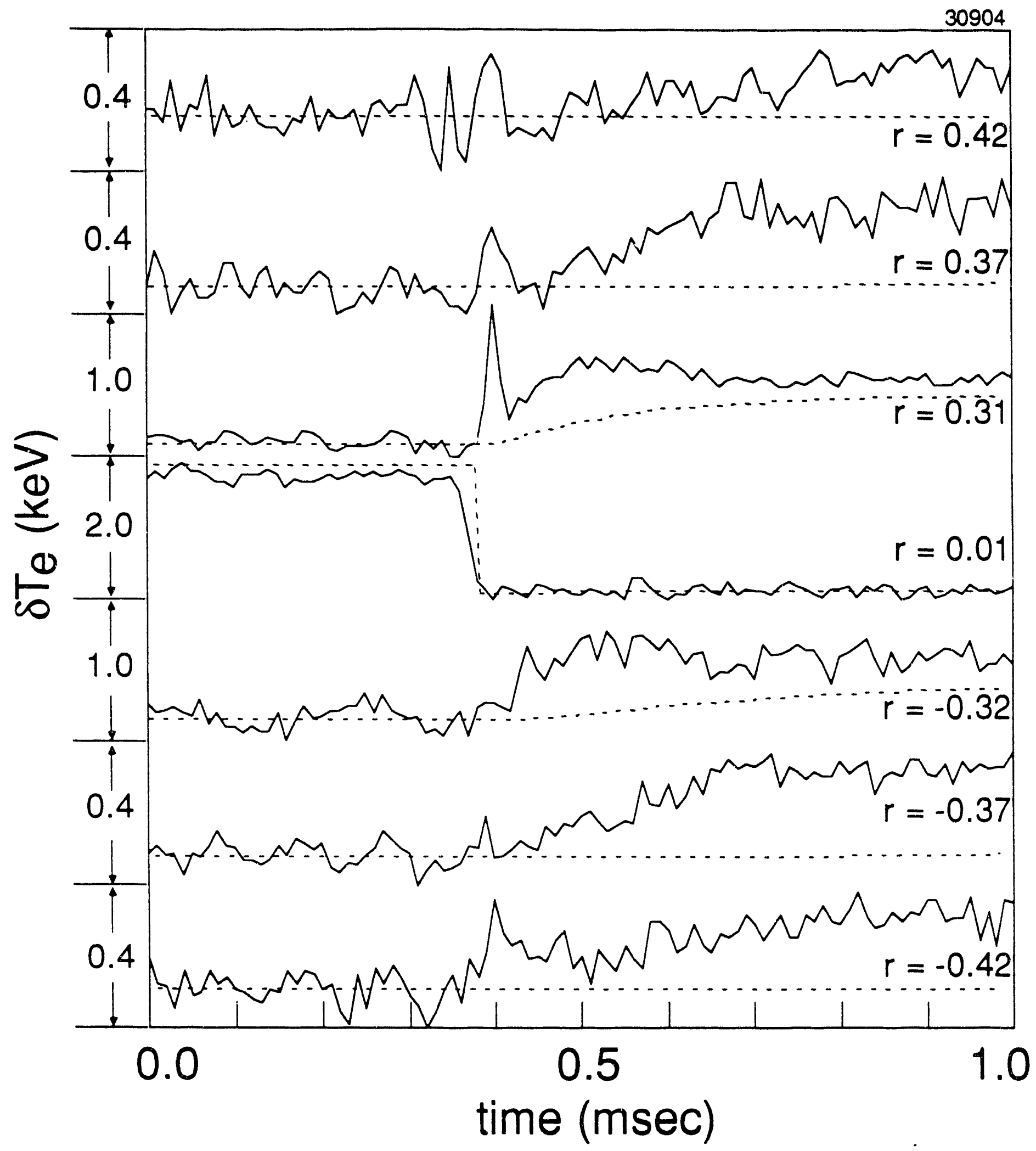

Figure 8 


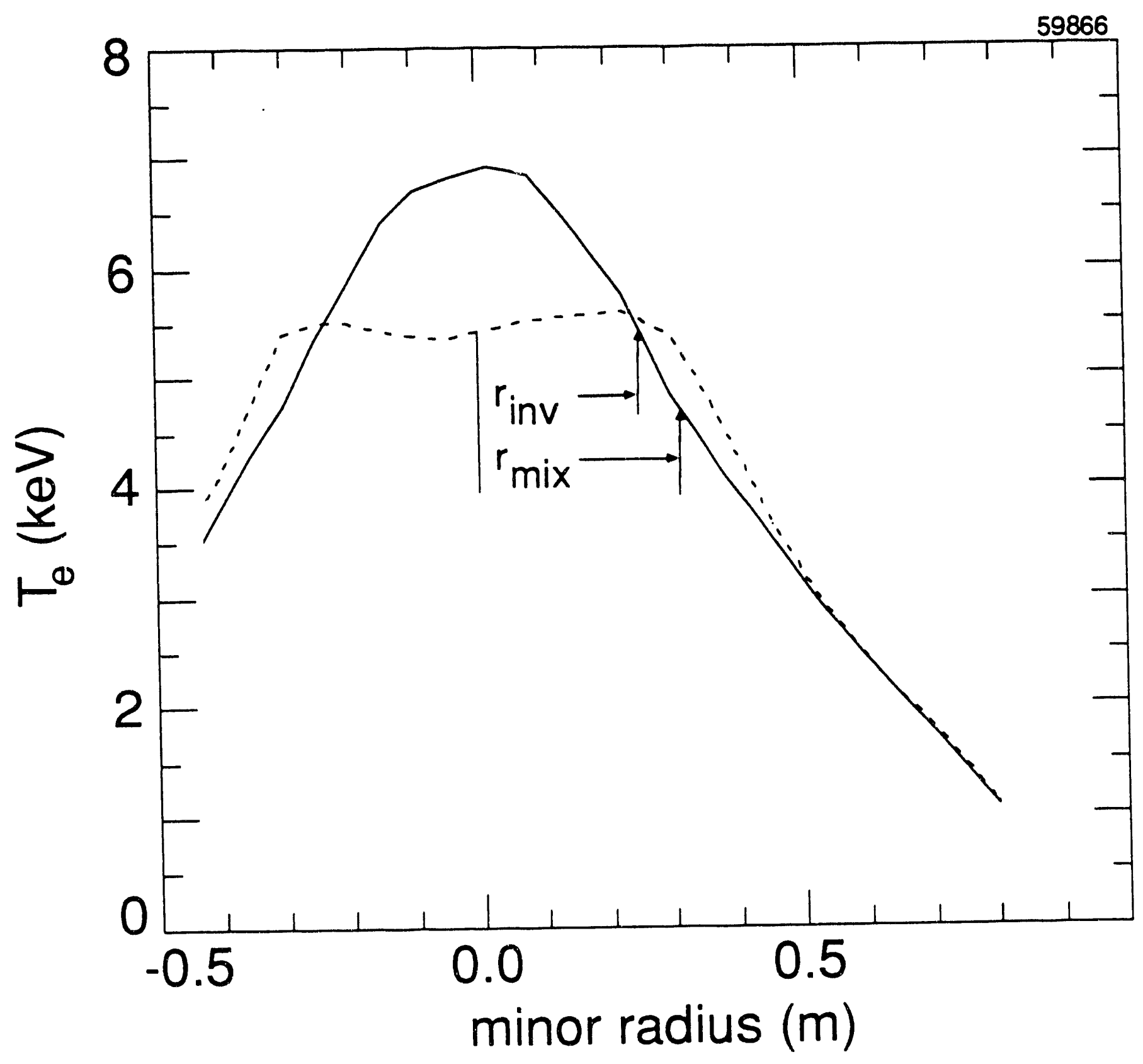




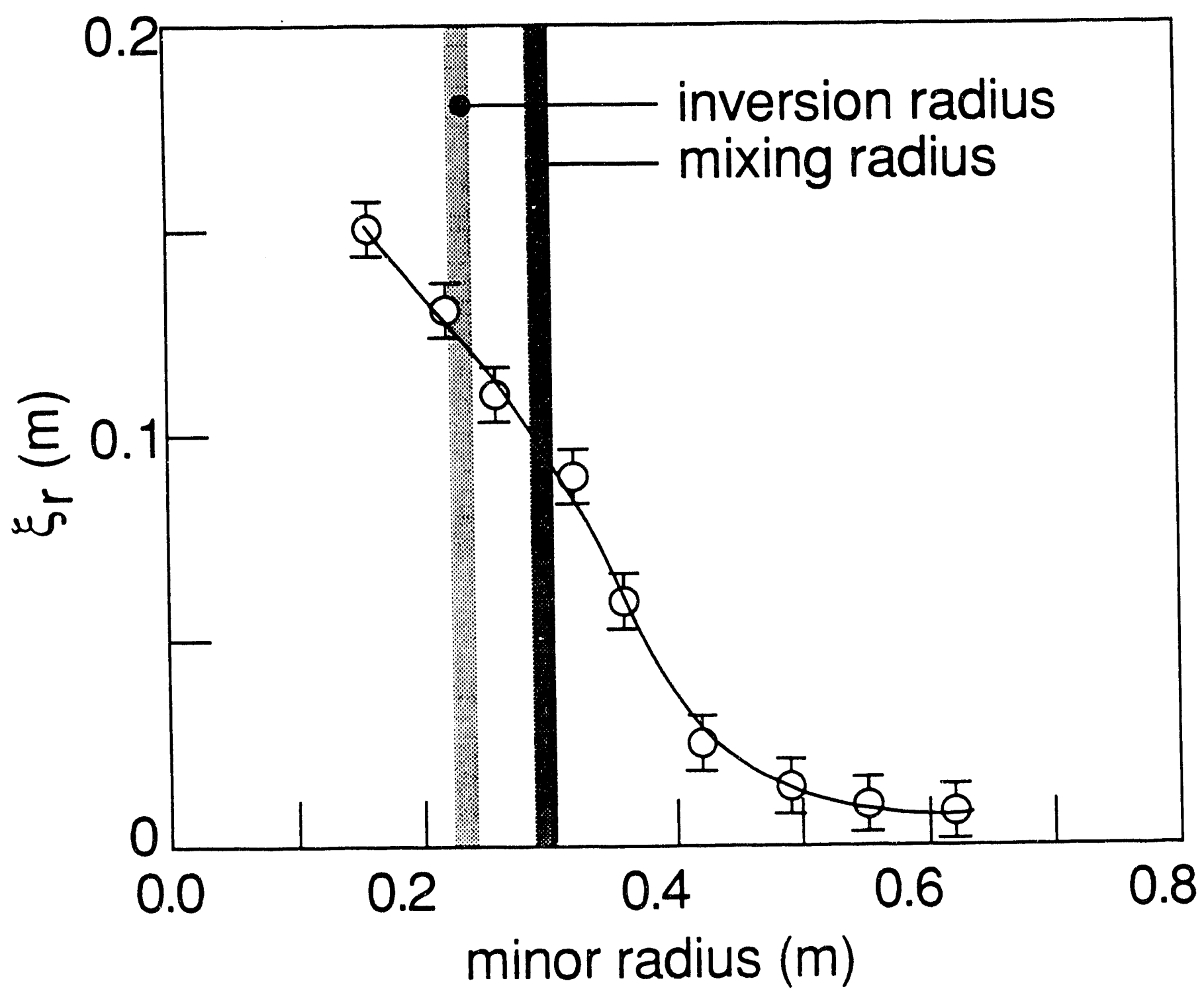

Figure 10 


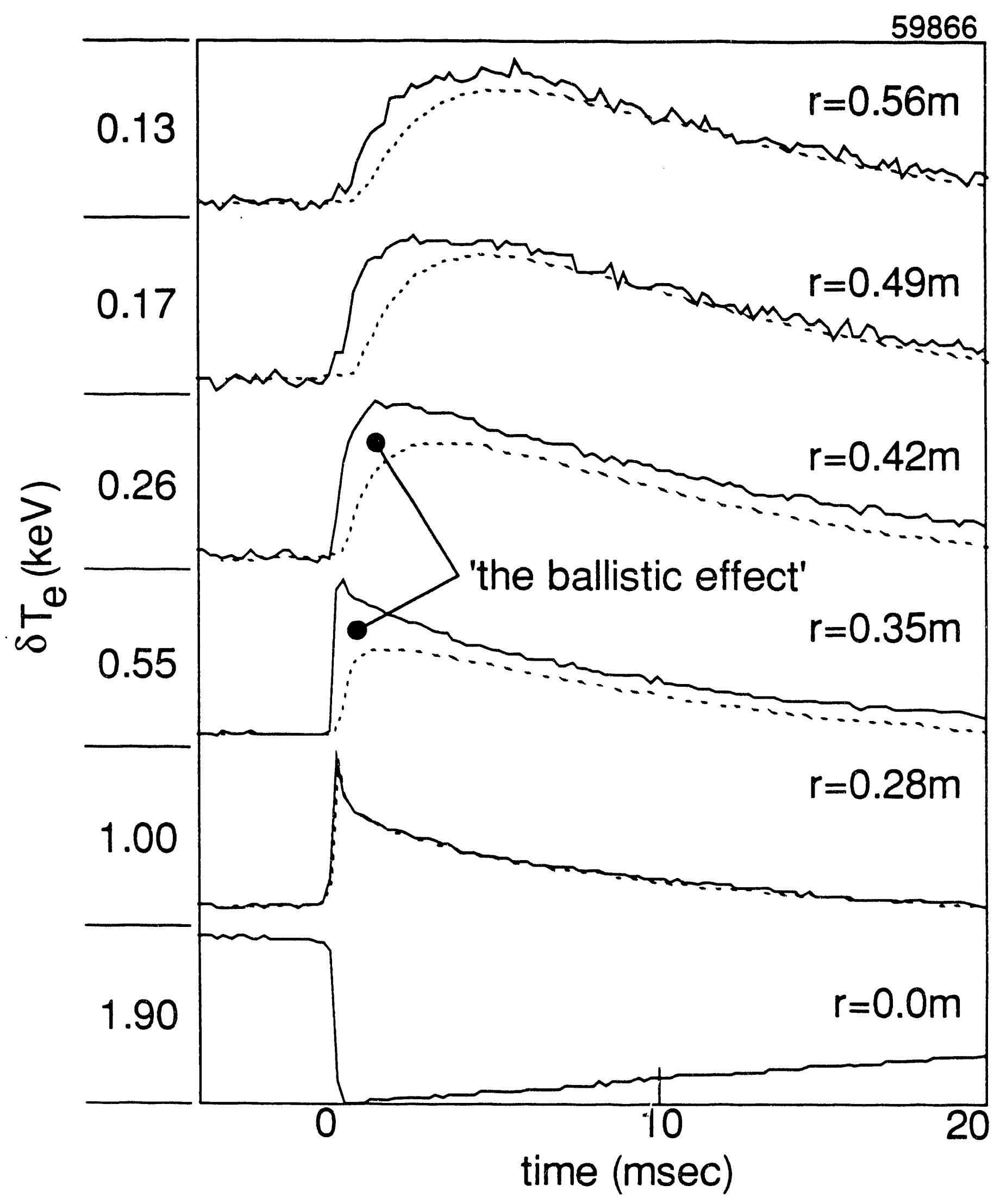




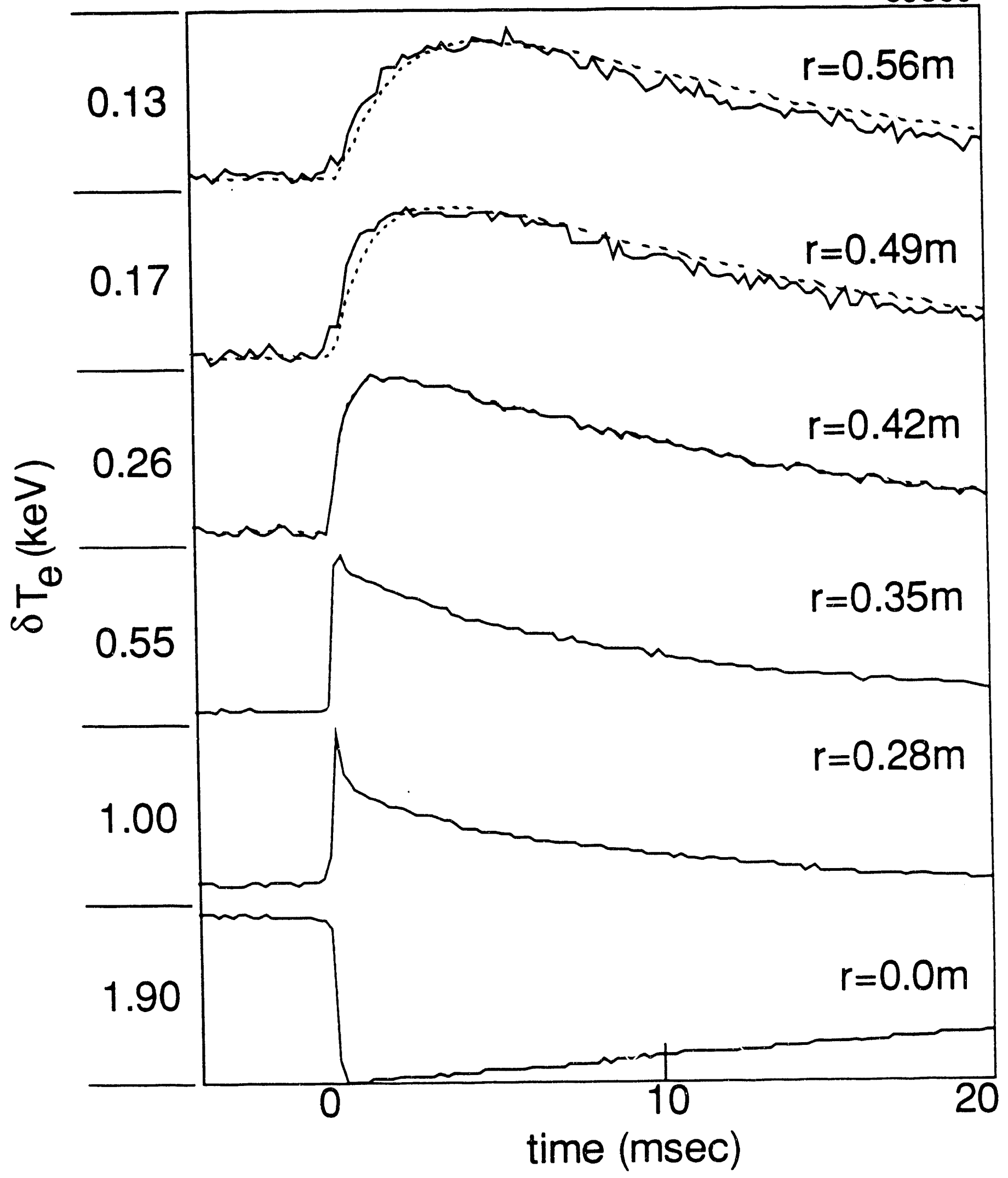




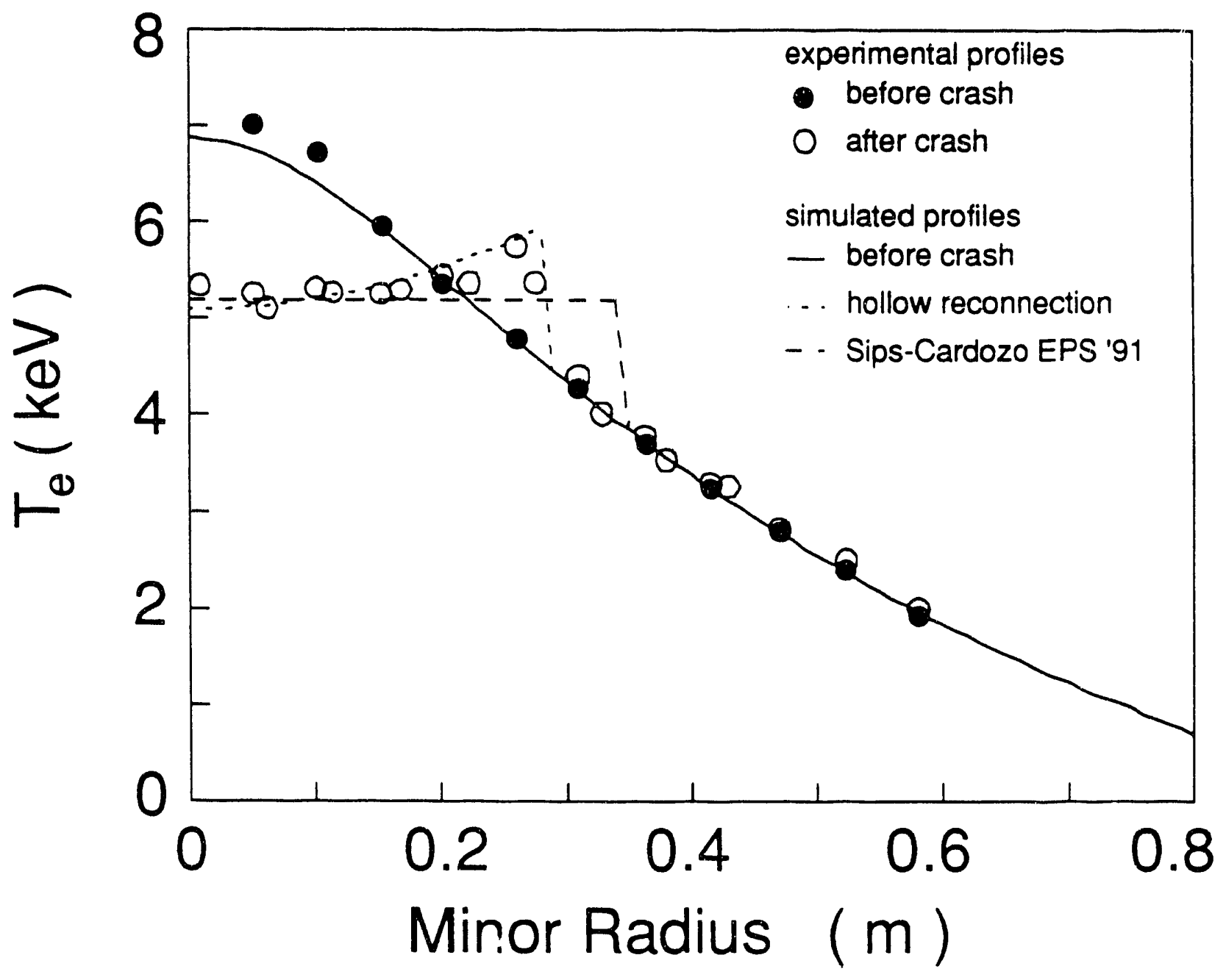

Figure 12 


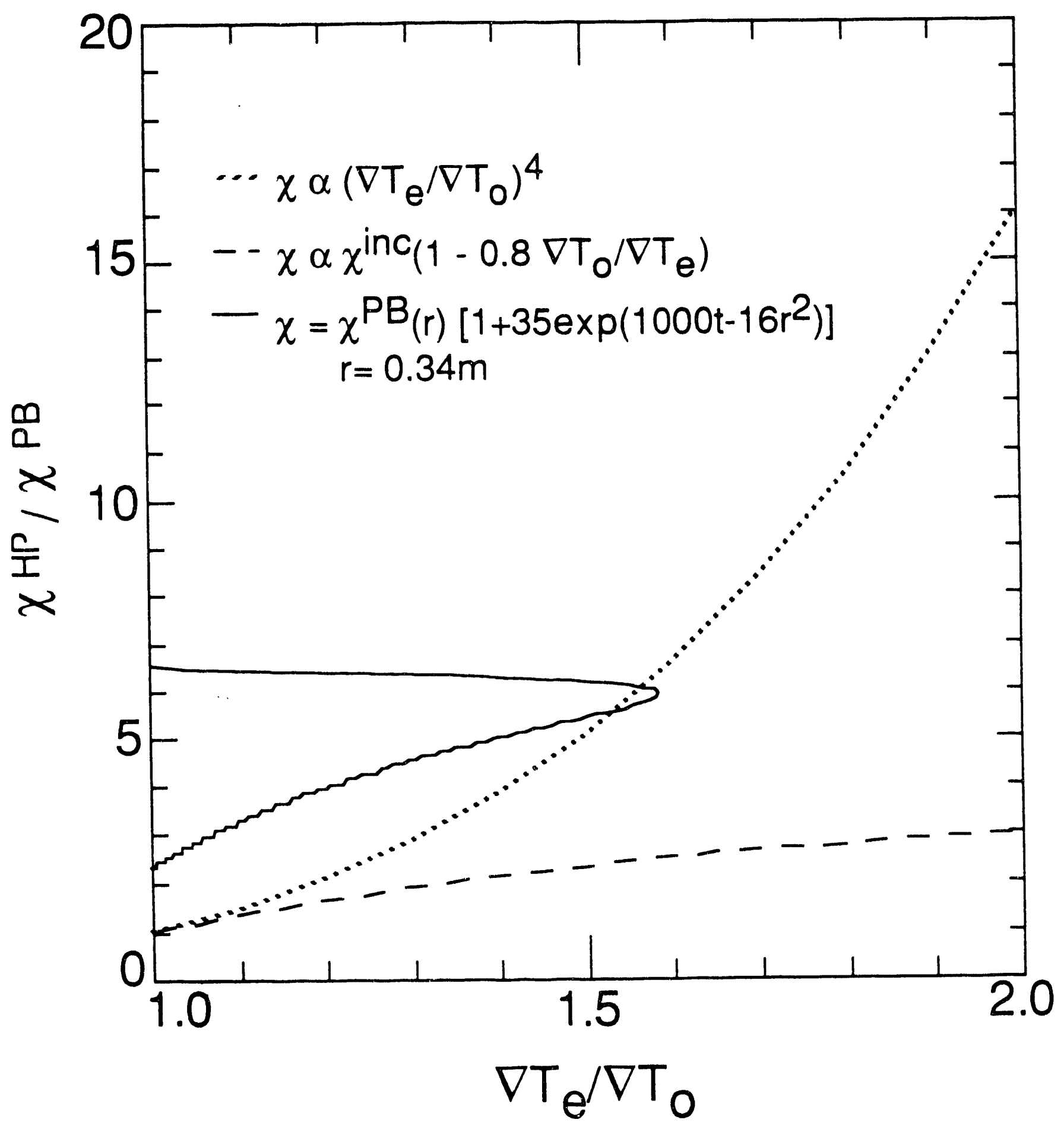

Figure 13 


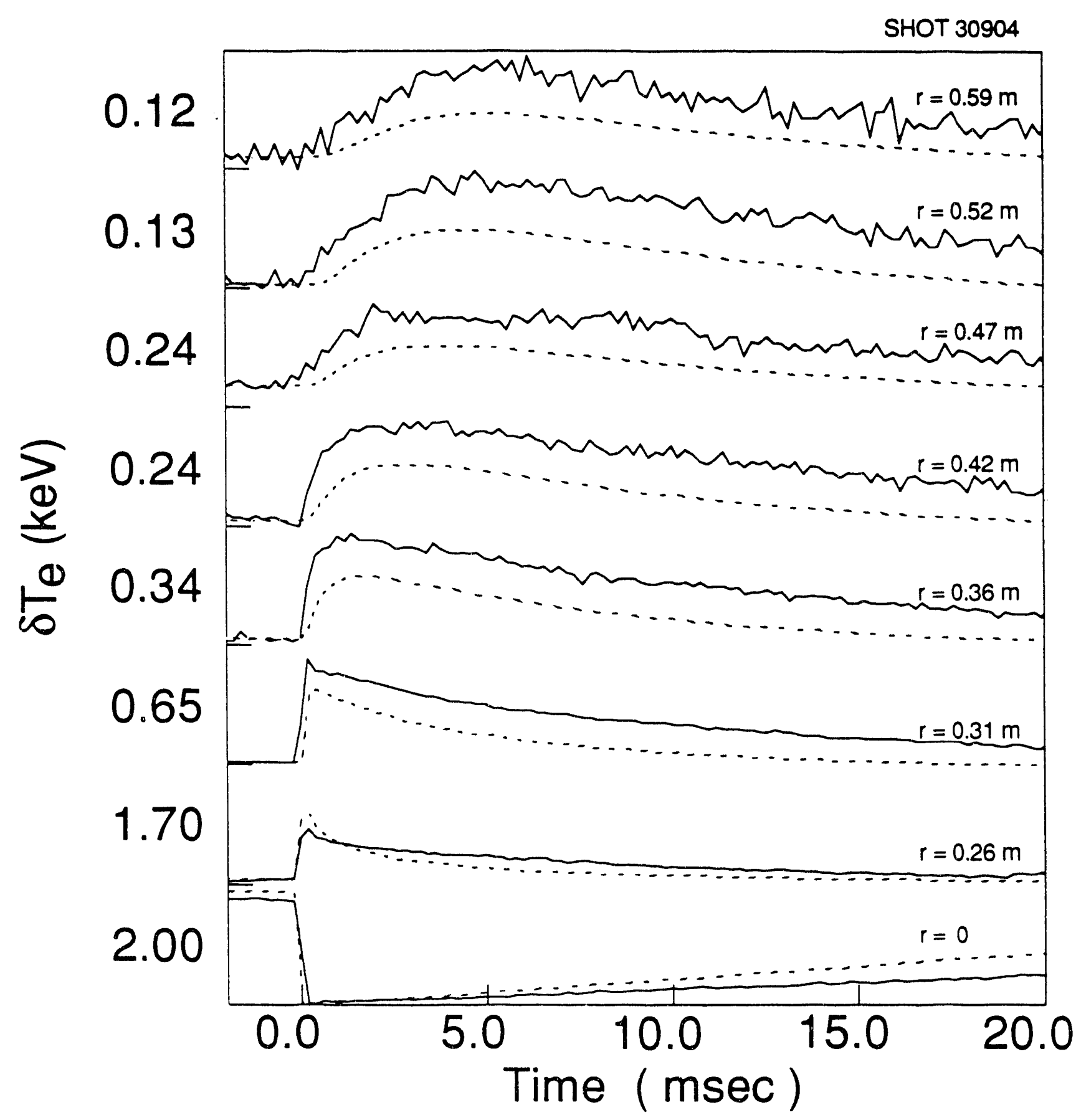

Figure 14 
SHOT 30904

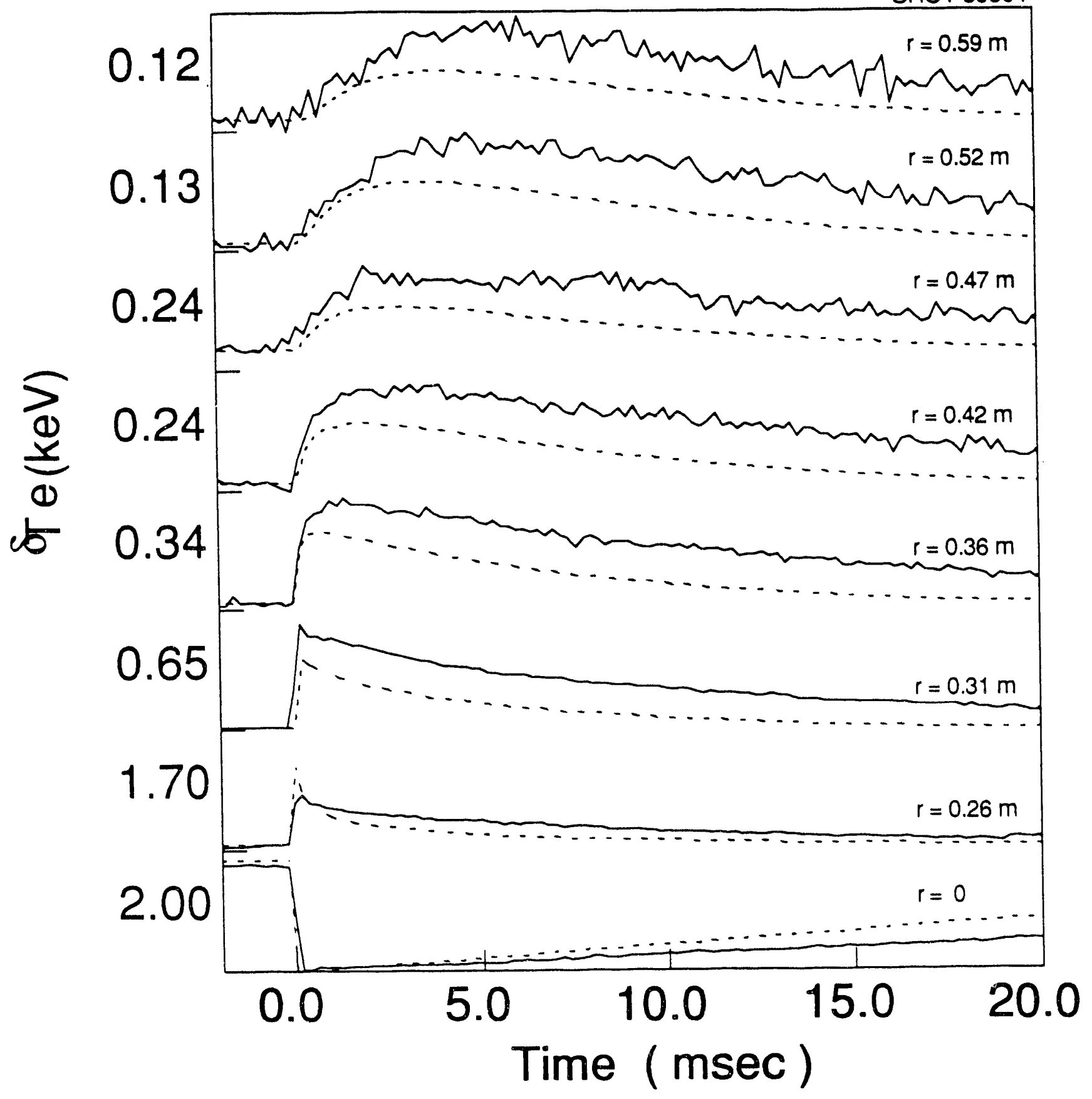




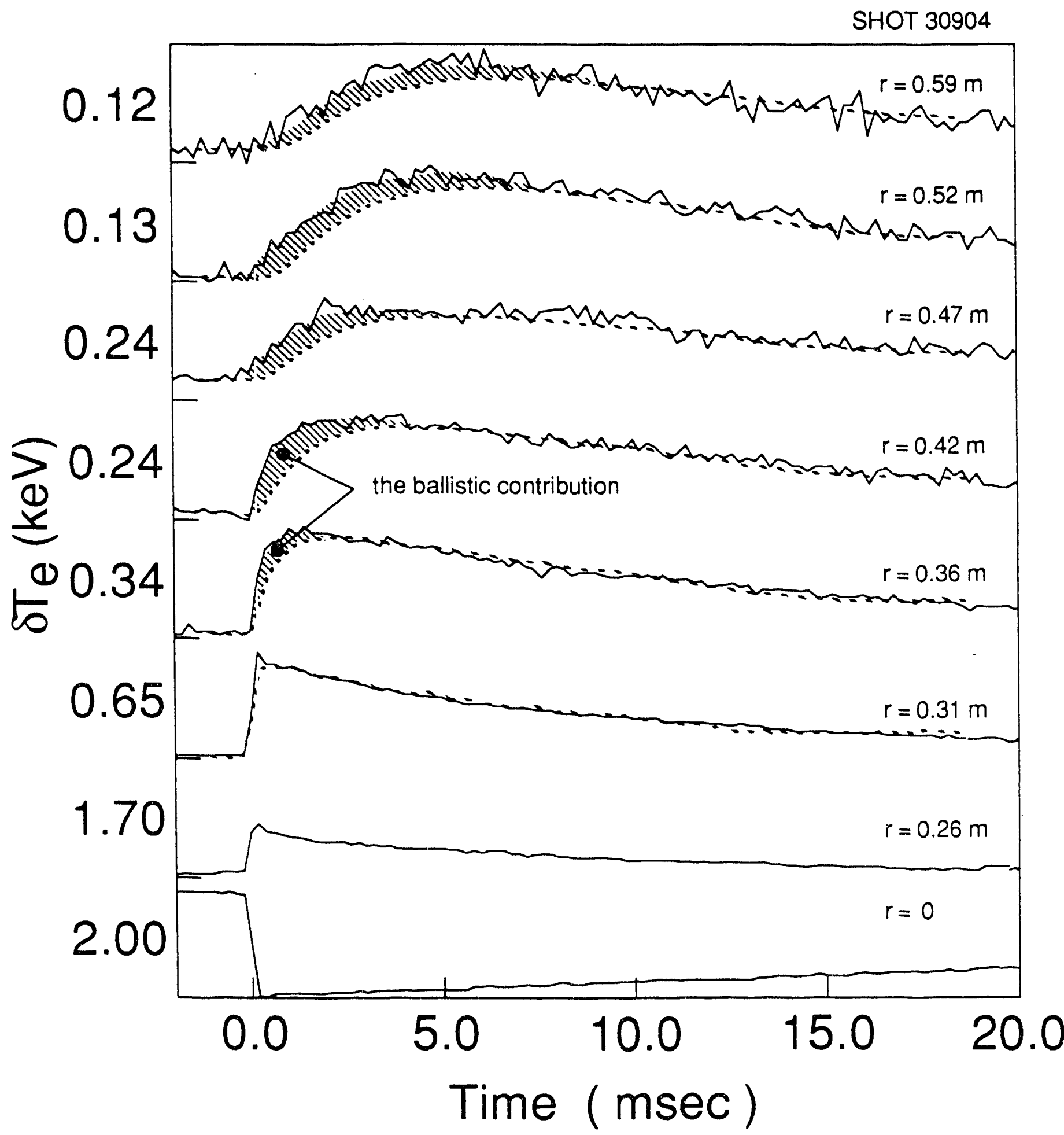


SHOT 30904

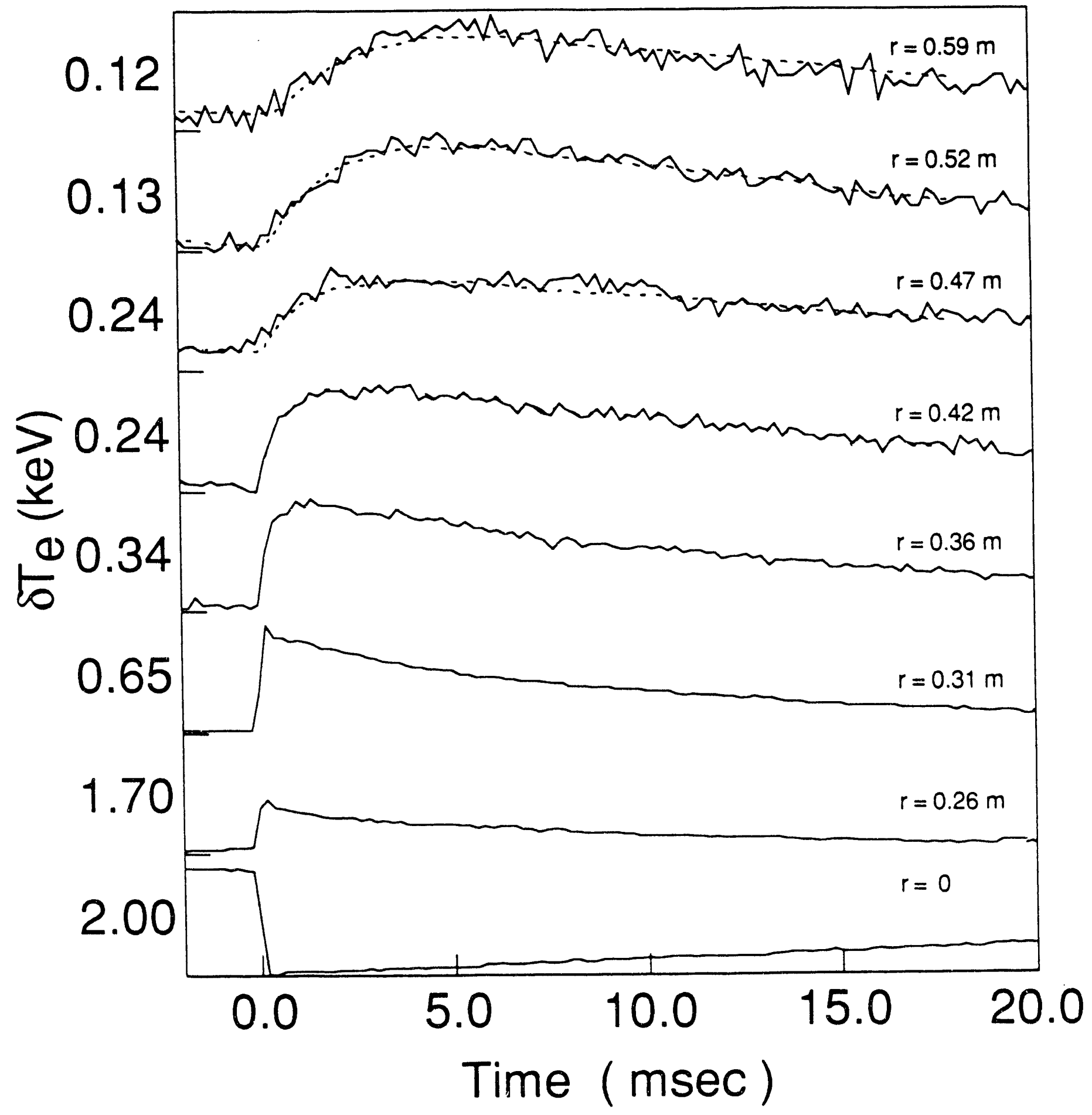

Figure 16b 


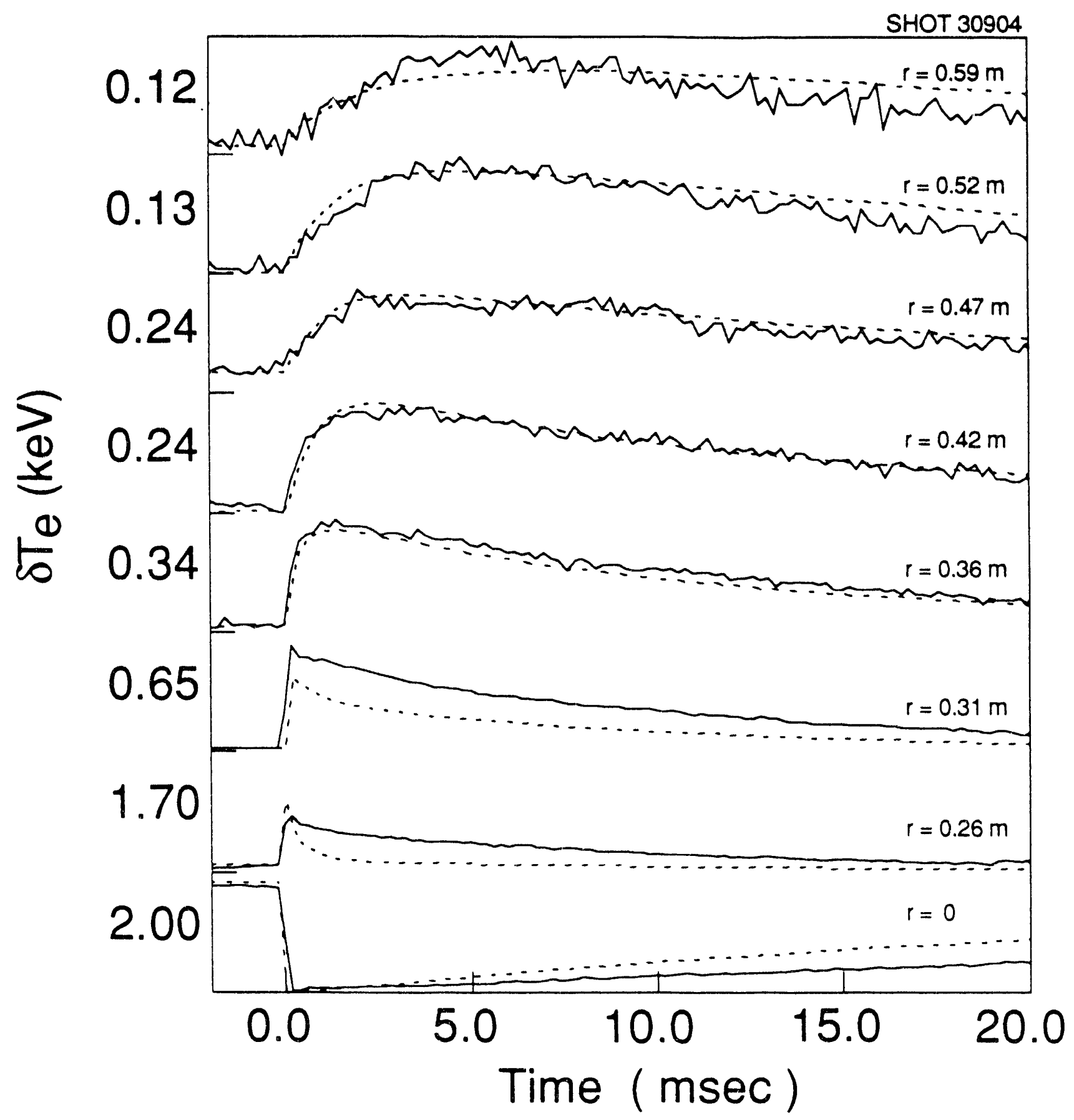

Figure 17 


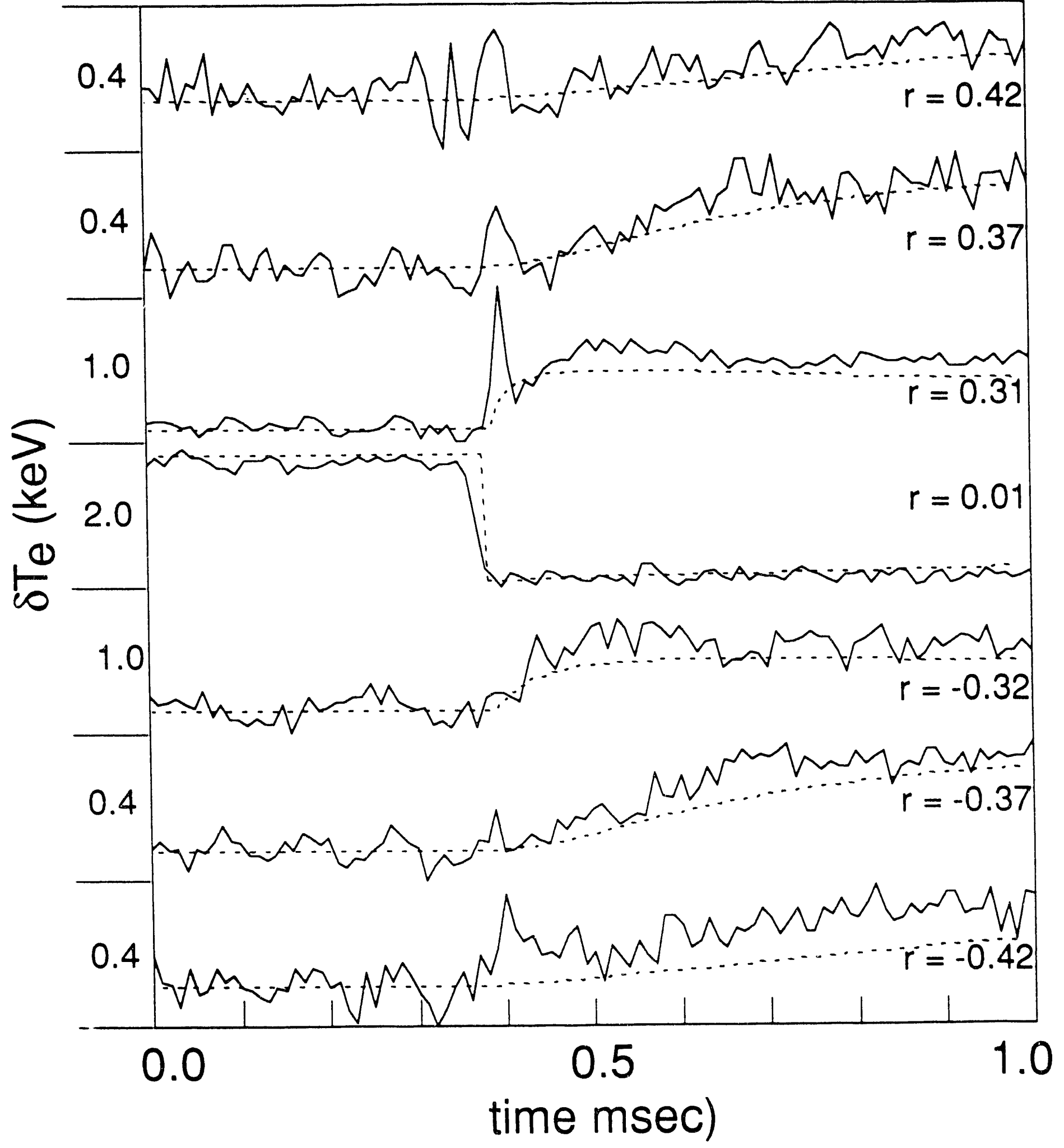

Figure 18 
Dr. F. Peoboni. Univ. of Wollongong. AUSTRALIA

Prof. M.X. Brennan, Univ. of Sydnoy, AUSTRALIA

Plasma Research Lab., Austration Nal Univ., AUSTRALIA

Prot. I.F. Jonses. Flindors Univ, AUSTRALIA

Prof. F. Cep. Inst for Theoretical Physics, AUSTRIA

Prot. M. Heinder, Institut for Thoorobiec \& Physik, AUSTRIA

Prof. M. Gooseons, Astronomiect Institurt BEI SIUM

Ecodo Royedo Mliting, Leb. do Phy. Plaemas, BELGIUM

Commiscion-Europeen. DG. XII-Fusion Prog., BELGIUM

Prof. R. Bouciquh. Ritkeuriveritin Gent, BELGIUM

Dr. P.H. Sakeneka, Insideto Frica, BPUZIL

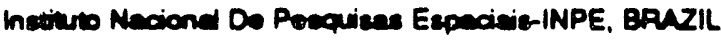

Documents Orion, Alomic Eneroy of Ceneda LU., CANADA

Dr. M.P. Bectynaki, MPB Technotogios, Inc., CANADA

Dr. H.M. Skengard, Univ. of Sackatchewen, CANADA

Prof. J. Teichmam, Univ. of Montreal, CANADE.

Prot. S.R. Sreenivean, Univ. of Cugary, CANADA

Prof. T.W. Johnetion. INRS-Energio. CANADA

Dr. A. Botion, Centre canedien de husion megnotique, CANADA

Dr. C.R. James.. Univ. of Nborta, CANADA

Dr. P. Luthe, Kom nekeno Univeredia, CZECHOSSLOVAKIA

The Librarien, Cuham Leboratory. ENGLAND

Librery. R61, Puthertord Appletion Leboratory. ENGLAND

Mrs. S.A. Hutchincon, JET Librey, ENGLAND

Dr. S.C. Sherma Univ. of South Pecific. FWI ISLANDS

P. Munonen, Univ. of Hedsiriti, FINLAND

Prof. M.N. Quesec, Ecol Patyochnique., FRANCE

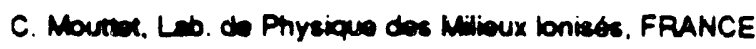

J. Alodel CENCADARACHE - Bat 506, FRANCE

Prot. E. Economou, Univ. of Crow, GREECE

Ms. C. Rimi, Univ. of loamina, GREECE

Dr. T. Muel, Acadamy Bibiographic Ser., HONG KONG

Proprint Librey, Hungarian Acadermy of Sa.. HUNGAPY

Dr. B. DesGupte, Saha inst of Nuctere Physics, INDIA

Or. P Kew, Inst. for Plasma Pesearch, INDIA

Dr. P. Rosenves, lereed Inst of Technology, ISPAEL

Liorarien, International Conter for Theo Physica, ITALY

Miss C. Do Pab. Associazions EURATOMENEA, ITALY

Dr. G. Groses. Istiuto of Fivica ded Plasma. ITALY

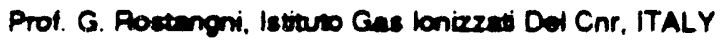

Dr. H. Yemedo. Toehibe Ras o Dend Center, JAPAN
Prof. I. Kowakemi, Hroshima Univ., JAPAN

Prof. K. Niehikawa, Hiroshima Univ., JAPAN

Director, Jepen Atomic Enorgy Posearch inst, JAPAN

Prof. S. Itoh, Kyustu Univ., JAPAN

Rosearch Into. Ct., National Instit. for Fusion Science. JAPAN

Prot. S. Tenake, Kyoso Univ., JAPAN

Librery, Kyob Univ., JAPAN

Prot. N. Inowe, Univ. of Tokyo, LAPAN

Socretary, Plemm Saction, Electrobchnical Lab., JAPAN

S. Mori, Technical Advieor, LAERI, JAPAN

Dr. O. Miture, Kumamots mat of Technology. JAPAN

J. Hyeon-Sook, Korea Alomic Enorgy Rosearch Inst. KOREA

D.I. Chai, The Korea Adv. Inst of Sai. \& Tech., KOREA

Prof. B.S. Liby. Univ. of Waikato. NEW ZEALAND

Inat of Phycica, Chinaes Acad Sa PEOPLE'S REP. OF CHINA

Librey. Inat of Plasma Physics, PEOPLE'S REP. OF CHINA

Tringhue Univ. Liorey, PEOPLE'S REPUBLIC OF CHINA

$Z$ Li, S.W. Inat Physica. PEOPLE'S REPUBUC OF CHINA

Prof. J.A.C. Gebre, Instibuto Superior Tecrico, PORTUGAL

Dr. O. Potrus, AlI CUzA Univ., ROMANIA

Dr. J. Viliers, Fusion Studies, AEC, S. AFRICA

Prof. M.A. Hellberg, Univ. of Natal, S. AFRICA

Prof. D.E. Km, Pohang Inet. of Sa. \& Tech., SO. KOREA

Prot. C.I.E.M.A.T, Fuaion Division Library, SPAIN

Dr. L Simtlo, Univ. of UMEA, SWEDEN

Lbrery, Royd inet of Tectrology, SWEDEN

Prot. H. Whatmson, Chalmers Univ. of Tech., SWEDEN

Contre Phys. Dos Plasmas, Ecolo Potrect. SWITZERLAND

Bibliotheak. Inst. Voor Plusma-Fysica, THE NETHERLANDS

Aest Prot. Dr. S. Cekir, Middo East Tech. Univ. TURKEY

Dr. V.A. Gukhikh, Sci. Ros. Inst Eloctrophys.I Appantus, USSR

D. D.D. Ayutov, Siberian Branch of Acadomy of Sa., USSR

Or. G.A. Eiseev, I.V. Kurchatov Inst, USSR

Librevien, The Ukr.SSR Acaderny of Scionces. USSR

Or. LM. Kovizhnykh, Inet. of Gonord Physics. USSP

Komtorectungeanlege GmbH, Zentrabibliothek, W. GERMANY

Bibliothat Inst For Plasmatorschung, W. GERMANY

Prot. K. Schinder, Rutr-Universit Bochum, W. GERMANY

Or. F. Weoner, (ASOEX), Max-Planck-Institust W. GERMANY

Librerion, Maxplench-Institur, W. GERMANY

Prof. R.K. Janew, Inat of Plyysica, YUcosLAVIA 

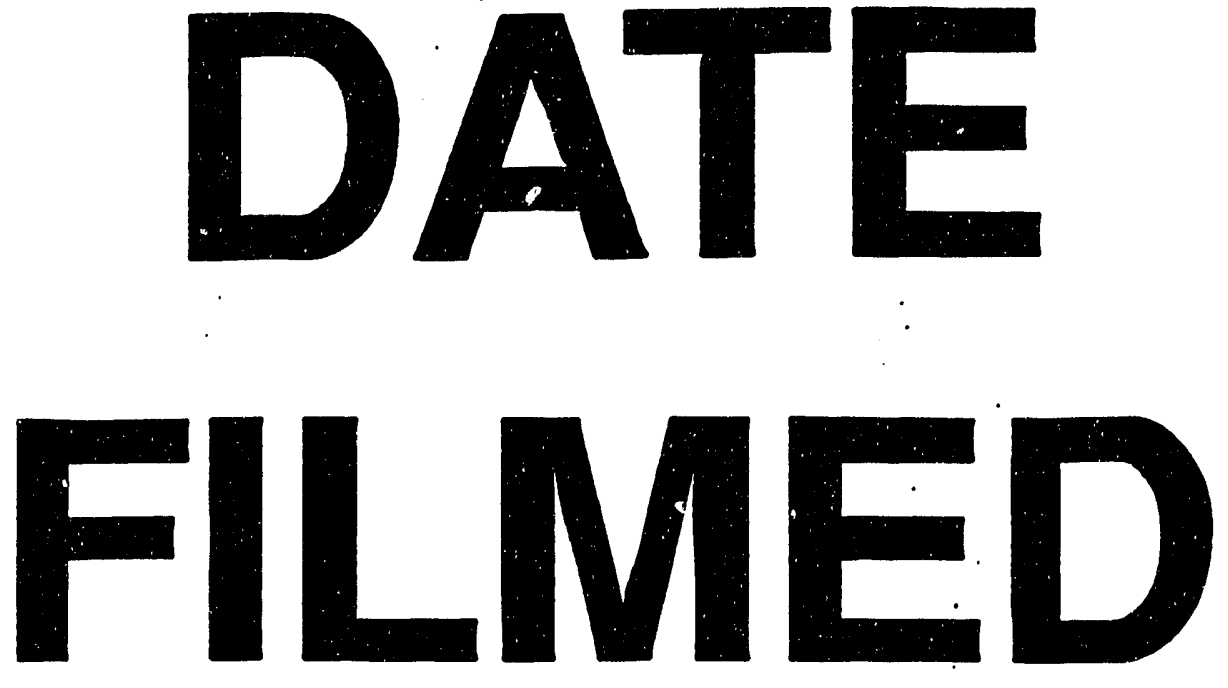

$10 / 20 / 93$
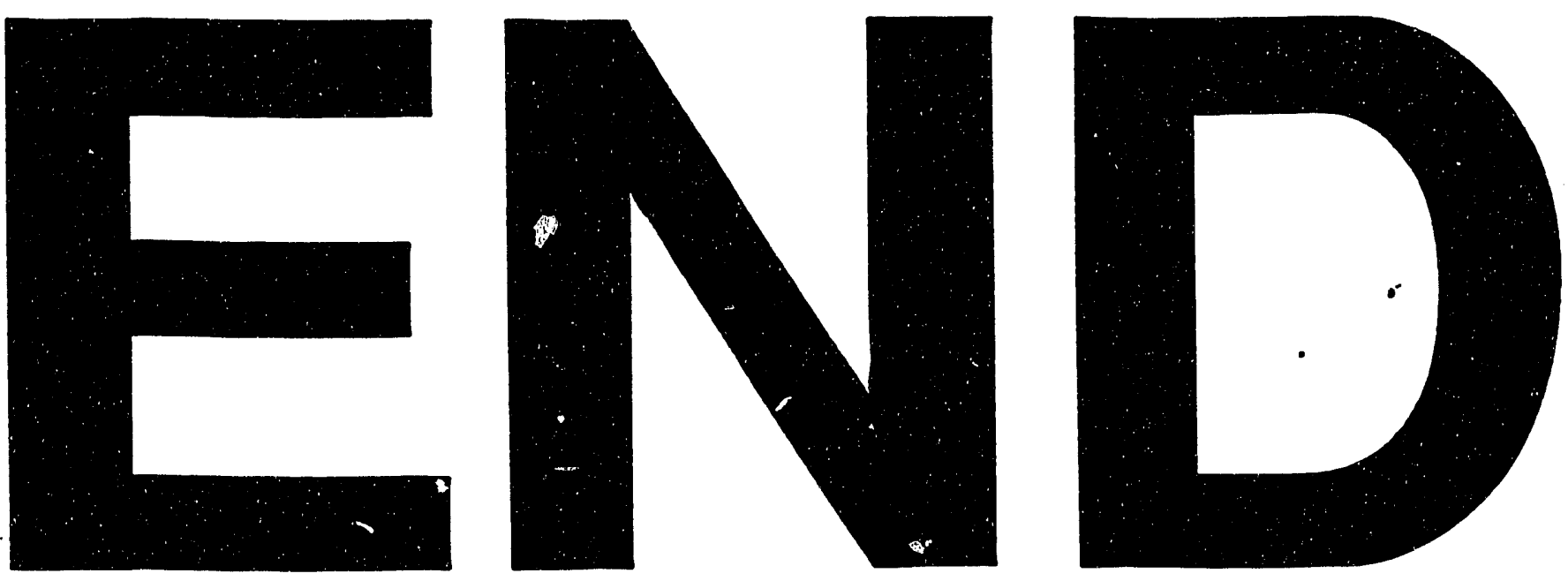
